\title{
Search for Scalar Leptons, Charginos and Neutralinos in $\mathrm{e}^{+} \mathrm{e}^{-}$collisions at $\sqrt{s}=161-172 \mathrm{GeV}$
}

The L3 Collaboration

\begin{abstract}
Scalar leptons, charginos and neutralinos, predicted by supersymmetric theories, have been searched for in data samples collected with the L3 detector at centre-ofmass energies of $161 \mathrm{GeV}$ and $172 \mathrm{GeV}$ with integrated luminosities of $10.7 \mathrm{pb}^{-1}$ and $10.0 \mathrm{pb}^{-1}$ respectively. No evidence for such particles is found. New limits on production cross sections are set, assuming R-parity conservation. New exclusion contours in the Minimal Supersymmetric Standard Model parameter space as well as new lower limits on masses are derived.
\end{abstract}

Submitted to Phys. Lett. B 


\section{Introduction}

One of the main objectives of the LEP experiments is the search for supersymmetric particles (SUSY) predicted in theories beyond the Standard Model [1]. They are partners of the known particles with a spin difference of one half. In addition supersymmetric models require at least two Higgs doublets to generate the masses of the gauge bosons and of the fermions.

In this letter the following assumptions are made: $\mathrm{R}$-parity, a quantum number which distinguishes ordinary particles from supersymmetric particles, is conserved and the neutralino $\left(\tilde{\chi}_{1}^{0}\right)$ is the lightest supersymmetric particle. The conservation of $\mathrm{R}$-parity implies that supersymmetric particles are produced in pairs and decay into non-supersymmetric particles plus the $\tilde{\chi}_{1}^{0}$ which is stable and escapes detection due to its weakly interacting nature.

Scalar leptons $\left(\tilde{\ell}_{R}^{ \pm}\right.$and $\left.\tilde{\ell}_{L}^{ \pm}\right)$are the supersymmetric partners of the right- and left-handed charged leptons. They are produced in pairs through $s$-channel $\gamma / \mathrm{Z}$ exchange. The production of scalar electrons receives contributions also from the $t$-channel exchange of a neutralino which enhances the production cross section. The scalar lepton decays into its associated lepton mainly via $\tilde{\ell}^{ \pm} \rightarrow \tilde{\chi}_{1}^{0} \ell^{ \pm}$.

Charginos $\left(\tilde{\chi}_{1}^{ \pm}\right)$are pair-produced via $s$-channel $\gamma / Z$ or $t$-channel scalar neutrino $(\tilde{\nu})$ exchange. When the masses of the charged scalar leptons $\left(\tilde{\ell}^{ \pm}\right)$, the scalar neutrino and the charged Higgs bosons $\left(\mathrm{H}^{ \pm}\right)$are very large, the $\tilde{\chi}_{1}^{ \pm}$decays via exchange of a virtual $\mathrm{W}^{*}$ : $\tilde{\chi}_{1}^{ \pm} \rightarrow \tilde{\chi}_{1}^{0} \mathrm{~W}^{*} \rightarrow \tilde{\chi}_{1}^{0} f \bar{f}^{\prime}$. If the $\tilde{\ell}^{ \pm}$and $\tilde{\nu}$ masses are comparable to $M_{\mathrm{W}}$ the chargino also decays via virtual scalar lepton or scalar neutrino exchange and the leptonic branching fraction is enhanced. Finally for $\tilde{\ell}^{ \pm}$and $\tilde{\nu}$ lighter than the chargino, the decay modes $\tilde{\chi}_{1}^{ \pm} \rightarrow \tilde{\ell}^{ \pm} \nu$ or $\tilde{\chi}_{1}^{ \pm} \rightarrow \tilde{\nu} \ell^{ \pm}$become dominant.

Neutralino pair production $\mathrm{e}^{+} \mathrm{e}^{-} \rightarrow \tilde{\chi}_{i}^{0} \tilde{\chi}_{j}^{0}(i, j=1, \ldots, 4$; ordered by their masses $)$ proceeds via $s$-channel $\mathrm{Z}$ or $t$-channel scalar electron $\left(\tilde{\mathrm{e}}^{ \pm}\right)$exchange. We distinguish two classes of detectable processes: $\mathrm{e}^{+} \mathrm{e}^{-} \rightarrow \tilde{\chi}_{1}^{0} \tilde{\chi}_{2}^{0}$ and $\mathrm{e}^{+} \mathrm{e}^{-} \rightarrow \tilde{\chi}_{2}^{0} \tilde{\chi}_{2}^{0}$. The decays of heavier neutralinos provide similar experimental signatures. When the masses of neutral SUSY Higgs bosons (h, $\mathrm{A})$, the $\tilde{\ell}^{ \pm}$and the $\tilde{\nu}$ are very large, the heavier neutralinos $\left(\tilde{\chi}_{j}^{0}, j \geq 2\right)$ decay via virtual $\mathrm{Z}^{*}$ exchange $\tilde{\chi}_{j}^{0} \rightarrow \tilde{\chi}_{k}^{0} \mathrm{Z}^{*} \rightarrow \tilde{\chi}_{k}^{0} f \bar{f}$ with $k<j$. For a chargino lighter than neutralinos the latter decay via virtual $\mathrm{W}^{*}$ exchange $\tilde{\chi}_{j}^{0} \rightarrow \tilde{\chi}_{1}^{ \pm} f \bar{f}^{\prime}$. If the $\tilde{\nu}$ and $\tilde{\ell}^{ \pm}$masses are comparable to the $\mathrm{Z}$ mass, the neutralino decays also via a virtual scalar lepton, enhancing the leptonic branching fraction. Finally, for $\tilde{\nu}$ and $\tilde{\ell}^{ \pm}$lighter than neutralinos the two-body decays $\tilde{\chi}_{j}^{0} \rightarrow \tilde{\ell}^{ \pm} \ell^{\mp}$ or $\tilde{\chi}_{j}^{0} \rightarrow \tilde{\nu} \nu(\mathrm{j} \geq 2)$ become dominant. The radiative decays $\tilde{\chi}_{j}^{0} \rightarrow \tilde{\chi}_{k}^{0} \gamma$ are also possible via higher-order diagrams.

Limits on the existence of supersymmetric particles have been obtained by L3 [2] and other LEP experiments [3], as well as Tevatron experiments [4]. In what follows results are presented of a search for scalar leptons, charginos and neutralinos at centre-of-mass energies of 161 and $172 \mathrm{GeV}$ with the L3 experiment. Limits are presented on the production cross sections of SUSY particles and on parameters of the Minimal Supersymmetric Model (MSSM) [5]. To obtain these limits the new data are combined with our results previously obtained at lower centre-of-mass energies $[2,6,7]$.

\section{Data Sample and Simulation}

Here we present the analysis of the data collected by the L3 detector [8] during the high energy runs of LEP in 1996, corresponding to an integrated luminosity of $10.7 \mathrm{pb}^{-1}$ at $\sqrt{s}=161.3 \mathrm{GeV}$, 
$1.0 \mathrm{pb}^{-1}$ at $\sqrt{s}=170.3 \mathrm{GeV}$ and $9.0 \mathrm{pb}^{-1}$ at $\sqrt{s}=172.3 \mathrm{GeV}$, referred to as $161 \mathrm{GeV}$ and 172 $\mathrm{GeV}$ data.

Monte Carlo generators are used to simulate events from the following reactions: PYTHIA [9] for $\mathrm{e}^{+} \mathrm{e}^{-} \rightarrow \mathrm{q} \overline{\mathrm{q}}, \mathrm{e}^{+} \mathrm{e}^{-} \rightarrow \mathrm{Ze}^{+} \mathrm{e}^{-}$and $\mathrm{e}^{+} \mathrm{e}^{-} \rightarrow \gamma / \mathrm{Z} \gamma / \mathrm{Z}$; EXCALIBUR [10] for $\mathrm{e}^{+} \mathrm{e}^{-} \rightarrow \mathrm{W}^{ \pm} \mathrm{e}^{\mp} \nu$; KORALZ [11] for $\mathrm{e}^{+} \mathrm{e}^{-} \rightarrow \mu^{+} \mu^{-}$and $\mathrm{e}^{+} \mathrm{e}^{-} \rightarrow \tau^{+} \tau^{-}$; BHAGENE3 [12] for $\mathrm{e}^{+} \mathrm{e}^{-} \rightarrow \mathrm{e}^{+} \mathrm{e}^{-}$; KORALW [13] for $\mathrm{e}^{+} \mathrm{e}^{-} \rightarrow \mathrm{W}^{+} \mathrm{W}^{-}$; two-photon interaction processes have been simulated using DIAG36 [14] $\left(\mathrm{e}^{+} \mathrm{e}^{-} \rightarrow \mathrm{e}^{+} \mathrm{e}^{-} \ell^{+} \ell^{-}\right)$and PHOJET [15] ( $\mathrm{e}^{+} \mathrm{e}^{-} \rightarrow \mathrm{e}^{+} \mathrm{e}^{-}$hadrons), requiring at least $3 \mathrm{GeV}$ for the invariant mass of the two-photon system. The number of simulated events for each background process is equivalent to more than 100 times the statistics of the collected data sample except for two-photon interactions for which it is more than three times the data statistics.

Signal events have been generated with the Monte Carlo program SUSYGEN [16], for masses of SUSY particles $\left(M_{s p}\right)$ ranging from $45 \mathrm{GeV}$ up to the kinematic limit and for $\Delta \mathrm{M}$ values $\left(\Delta M=M_{s p}-M_{\tilde{\chi}_{1}^{0}}\right)$ between $3 \mathrm{GeV}$ and $M_{s p}-1 \mathrm{GeV}$. In the case of the chargino and neutralino analyses we simulate for each decay mode at least 1000 events per $\left(M_{s p}, \Delta M\right)$ point. Moreover, for charginos, events have also been generated with the program DFGT [17], which includes the spin correlation between charginos. The signal efficiencies obtained are in agreement between the two programs.

The detector response is modelled with the GEANT [18] program which includes the effects of energy loss, multiple scattering and showering in the detector materials and in the beam pipe. By means of randomly triggered events in coincidence with the beam crossing we estimate the amount of noise in the detector. We monitor the time-dependent detector behaviour and take into account detector inefficiencies in our analyses.

Hadronic events are reconstructed using information from all sub-detectors. The energy of the event is obtained from the energy depositions in the electromagnetic and hadronic calorimeters and the particle momenta as measured in the central tracking and muon chambers.

An electron is identified as an electromagnetic shower matched with a track. An electromagnetic shower not matched with a track is identified as a photon. The energy measured in the electromagnetic calorimeter has to be larger than $1 \mathrm{GeV}$ and is assigned to the identified electron or photon. A muon is identified by a track in the muon chambers which is matched with a track in the central chamber. Taus are defined as isolated hadronic narrow jets with energy larger than $2 \mathrm{GeV}$ and one to three associated tracks. The energy of the tau is defined as the energy contained in a cone of $10^{\circ}$ half opening angle around the tau direction. Its isolation is assured by requiring that there are no additional tracks and no more than two additional calorimetric clusters in a cone of $30^{\circ}$ half opening angle and that the ratio of the energies in the two cones is less than 2.0 .

Remaining clusters and tracks are classified as hadrons. Jets are reconstructed with the Durham algorithm [19] forcing the reconstruction into two jets.

\section{Analysis Procedure}

\subsection{Signal topologies and optimization procedure}

As explained in the introduction, we expect for all processes two undetected $\tilde{\chi}_{1}^{0}$ s in the final state. Therefore, the main characteristics of supersymmetric processes under study are large missing transverse momentum, missing energy, missing mass and acoplanarity. We apply three types of selection criteria oriented to all possible decays of scalar leptons, charginos and neutralinos, as listed below: 
- topology 1: at least two acoplanar leptons;

- topology 2: hadrons and at least one isolated lepton;

- topology 3: purely hadronic final state with high multiplicity.

To account for the three lepton types and for the different signatures of the SUSY particle considered in total, nine different selections are performed.

The signal topologies and the associated background sources depend strongly on $\Delta M$. Therefore all nine selections were optimized separately for three different $\Delta M$ ranges: the low $\Delta M$ range at $5-10 \mathrm{GeV}$, the medium $\Delta M$ at $20-30 \mathrm{GeV}$ and the large $\Delta M$ at $50-60 \mathrm{GeV}$. In the low $\Delta M$ range the expected topologies for the signal are characterized by a low multiplicity and a low visible energy. There the background is dominated by two-photon interactions. For large $\Delta M$ the signal signatures are very similar to those of $\mathrm{W}$-pair production.

As already reported in reference [2], the cut values are a priori optimized using Monte Carlo signal and background events. The optimization procedure varies all cuts simultaneously to maximize the signal efficiency and the background rejection. In practice, we maximize the sensitivity function $1 / \kappa$ which is related to the ratio between the average Poisson upper limit on the signal without background subtraction and the signal efficiency $\epsilon$ [20]

$$
\kappa=\sum_{n=0}^{\infty} k_{n} P_{b}(n) / \epsilon
$$

where $k_{n}$ is the $95 \%$ confidence level Poisson upper limit and $P_{b}(n)$ is the Poisson distribution for $n$ observed events with a background of $b$ events.

For a given signal, as explained above, the selections are optimized for particular $\Delta M$ values. For intermediate $\Delta M$ values, we consider the logical "OR" of the three different selections (low, medium, large $\Delta M)$ and the combination of selections giving the highest sensitivity, according to formula (1), is chosen. In this procedure, we take into account the overlap between the selected samples in data and Monte Carlo.

\subsection{Event selection}

Common to all selections is the rejection of tagged two-photon interactions. We require that the sum of the energies measured in the lead-scintillator ring calorimeter and in the luminosity monitors [8] is less than $10 \mathrm{GeV}$. These two detectors cover the polar angle range $1.5^{\circ}<\theta<9^{\circ}$ on both sides of the interaction point.

\subsubsection{Purely leptonic final states}

Given the low multiplicity of the signal, events are rejected if the number of tracks is larger than 6 or if the number of calorimetric clusters $\left(N_{c l}\right)$ is larger than 15 . We then require two or three identified leptons. The following quantities are defined: the energy depositions $\left(E_{25}^{\perp}\right.$ and $E_{25}$ ) within $\pm 25^{\circ}$ around the missing energy direction in the $\mathrm{R}-\Phi$ plane or in space respectively, and the energy deposition in a $60^{\circ}$ half opening angle cone around the vector opposite to the sum of the two jet directions in space $\left(E_{60}^{b}\right)$. When three leptons are identified, a cut on the sum of the three angles between the three lepton pairs $\left(\theta_{123}^{l}\right)$ is also applied. We also apply cuts on the lepton energy $\left(E_{l e p}\right)$, on the total transverse momentum of the leptons $\left(p_{\perp}\right)$, on their maximum acollinearity and acoplanarity, on the polar angle of the missing energy vector $\left(\theta_{\text {miss }}\right)$ and on the variable $E_{T T L}$. The latter is defined as the absolute value of the projection of 
the total momentum of the two leptons onto the direction perpendicular to the leptonic thrust computed in the R- $\phi$ plane. The cut values optimized for the scalar lepton searches, are quoted in Table 1 for the three $\Delta M$ ranges. As an example we show in Fig. 1a) the distribution of $E_{T T L}$ for identified purely leptonic final states. The data at $\sqrt{s}=172 \mathrm{GeV}$ are compared to the background Monte Carlo and to the expected signal $\mathrm{e}^{+} \mathrm{e}^{-} \rightarrow \tilde{\chi}_{1}^{+} \tilde{\chi}_{1}^{-}$with $M_{\tilde{\chi}_{1}^{ \pm}}=85 \mathrm{GeV}$ and $M_{\tilde{\chi}_{1}^{0}}=30 \mathrm{GeV}$.

\subsubsection{Lepton plus hadron final states}

We select events with at least one isolated electron, muon or tau for which the energy, not associated to the lepton, in a cone of $30^{\circ}$ half-opening angle around its direction is less than $2 \mathrm{GeV}$. We apply cuts on the number of tracks in the hadronic system $\left(N_{t k}-N_{l e p}\right)$ and the number of calorimetric clusters. Furthermore, cuts are applied on the missing energy direction isolation $\left(\theta_{\text {miss }}\right.$ and $\left.E_{25}^{\perp}\right)$, the total transverse momentum $\left(p_{\perp}\right)$, the energy of the isolated lepton $\left(E_{l e p}\right)$, the recoil mass $M_{r e c}$, as well as on the acoplanarity angle between the jet and the lepton. A cut is applied on the visible energy $\left(E_{v i s}\right)$ and $E_{T T J L}$ which is equivalent to $E_{T T L}$ using the momenta of the jet and the lepton. A cut on the invariant mass of the hadronic system $\left(M_{\text {had }}\right)$ removes the WW background.

The cut values are shown in Table 2 for the three $\Delta M$ ranges. The distributions of the visible energy and of the acoplanarity are shown in Figures 1b) and 1c) for events selected with looser cuts. The data at $\sqrt{s}=172 \mathrm{GeV}$ are compared to background Monte Carlo and to an $\mathrm{e}^{+} \mathrm{e}^{-} \rightarrow \tilde{\chi}_{1}^{+} \tilde{\chi}_{1}^{-}$signal with $M_{\tilde{\chi}_{1}^{ \pm}}=85 \mathrm{GeV}$ and $\Delta M=50 \mathrm{GeV}$.

\subsubsection{Purely hadronic final states}

The list of cuts is reported in Table 3 for the three $\Delta M$ ranges. Again, we apply cuts on $N_{c l}$, $N_{t k}, p_{\perp}, E_{v i s}$, acollinearity and acoplanarity as well as on the missing energy $\left(\theta_{\text {miss }}\right.$ and $E_{25}^{\perp}$, $\left.E_{25}\right)$. Other variables are the absolute value of the total momentum of the event along the beam line normalized to the visible energy $\left(p_{\|}\right)$, the recoil mass $\left(M_{\text {rec }}\right)$ and the visible mass $\left(M_{v i s}\right)$.

In the medium and large $\Delta M$ selections, a cut on the width of the two jets is applied. We define $y^{\perp}$ as the ratio between the scalar sum of the particle momenta transverse to the jet direction and the jet energy. This assures the selection of events with broad jets. In the low $\Delta M$ range a cut on the ratio $E_{T T J} / p_{\perp}$ is applied. $E_{T T J}$ is equivalent to $E_{T T L}$ using the momenta and the directions of the two jets. As an example the distribution of $p_{\perp}$ is shown in Figure 1d) for events selected with looser cuts. The data at $\sqrt{s}=172 \mathrm{GeV}$ are compared to background Monte Carlo and to an $\mathrm{e}^{+} \mathrm{e}^{-} \rightarrow \tilde{\chi}_{1}^{+} \tilde{\chi}_{1}^{-}$signal with $M_{\tilde{\chi}_{1}^{ \pm}}=85 \mathrm{GeV}$ and $\Delta M=50 \mathrm{GeV}$.

\subsection{Efficiencies and background contaminations}

The number of variables, used in the analyses, is reduced to one in the following manner: for a given set of ranges of cuts $X_{\text {loose }}^{i}$ and $X_{\text {tight }}^{i}$ (where $i=C u t_{1}, \ldots, C u t_{N}$ ) we define a variable $\xi$ which runs from 0 to 1 , and is linearly related to all the cut values such that when $\xi$ is 0 all the cuts are on the loose edge (many background events satisfy the selection) and when it is 1 all the cuts are on the tight edge (no or few background events pass the selection):

$$
X_{\text {cut }}^{i}=X_{\text {loose }}^{i}+\left(X_{\text {tight }}^{i}-X_{\text {loose }}^{i}\right) \times \xi .
$$


The data and Monte Carlo are compared as a function of the variable $\xi$. As illustrated in Figures 2a) and 2b) for the purely hadronic final states, the data and Monte Carlo simulations are essentially in agreement for the medium and the large $\Delta M$ selections while in the low $\Delta M$ range the background expectation coming from two-photon processes does not reproduce the data well, as can be seen in Figure 2c). The vertical arrows show the position of the optimized cuts. To avoid a dependence of the optimization on limited Monte Carlo statistics we use an analytical extrapolation of the background estimation (smooth curves in Figure 2).

For given values of SUSY particle and $\tilde{\chi}_{1}^{0}$ masses, and given decay modes, the combination of selections providing the highest sensitivity according to formula (1) is chosen. Each candidate and background event surviving this set of selections belongs to this $M_{s p}-M_{\tilde{\chi}_{1}^{0}}$ combination, without imposing any additional kinematic constraint. Some of these results are given in Tables 4 to 6 .

The selection efficiencies at $\sqrt{s}=161 \mathrm{GeV}$ and $\sqrt{s}=172 \mathrm{GeV}$ for scalar lepton masses ranging from 50 to $75 \mathrm{GeV}$, as well as the background expectations, are reported for different values of $\Delta M$ in Table 4. Efficiencies vary from $30 \%$ to $62 \%$ for scalar electrons and from $21 \%$ to $50 \%$ for scalar muons with a maximum background expectation of 0.7 events for each. In comparison, the scalar tau selection efficiencies are smaller.

Typical selection efficiencies, as well as the number of background events expected for a 80 $\mathrm{GeV}$ or $85 \mathrm{GeV}$ chargino mass for different decay channels and assuming a $100 \% \mathrm{~W}^{*} \tilde{\chi}_{1}^{0}$ decay mode, are displayed in Table 5. In the latter case, a maximum efficiency of $55 \%$ is reached for a background contamination of 0.3 events for $\Delta M=20 \mathrm{GeV}$. In the low $\Delta M$ region the efficiency decreases due to the large contamination of two-photon interactions and due to the lower trigger acceptance. For large $\Delta M$ it decreases because of the WW background. To extract the total number of expected background events, we take into account the overlap between the different selections.

The selection efficiencies, as well as the number of background events expected for a sum of neutralino masses $M_{\tilde{\chi}_{1}^{0}}+M_{\tilde{\chi}_{2}^{0}}=160 \mathrm{GeV}$ and $170 \mathrm{GeV}$ for different decay channels and assuming a $100 \% \mathrm{Z}^{*} \tilde{\chi}_{1}^{0}$ decay mode, are displayed in Table 6 . Compared to the chargino selection, the efficiencies are lower due to the invisible decays of the $Z^{*}$.

Systematic errors on the signal efficiencies are evaluated as for the data taken at $\sqrt{s}=$ $130-140 \mathrm{GeV}$ (see reference [2]). They are typically 5\% relative, dominated by Monte Carlo statistics.

\section{Results}

A summary of the searches at $\sqrt{s}=161 \mathrm{GeV}$ and $172 \mathrm{GeV}$ is given in Table 7 showing the number of candidates and expected background events. It should be noted that events can be selected by more than one selection. We do not observe any excess of events relative to what is expected from Standard Model processes.

Therefore, we set upper limits on the scalar lepton, chargino and neutralino production cross sections and limits on the masses of these particles in the framework of the MSSM. Exclusion limits at $95 \%$ C.L. are derived taking into account background contributions. As the background from two-photon processes is not well described by the Monte Carlo we conservatively do not include this contribution in the evaluation of the limits. To derive limits in the MSSM, we optimize the global selection for any different point in the parameter space. This is obtained, choosing every time the combination of selections providing the highest sensitivity, given the 
production cross sections and the decay branching fractions. The systematic errors are taken into account following the procedure explained in reference [21].

\subsection{Upper limits on scalar lepton, chargino and neutralino produc- tion cross sections}

To derive upper limits on the production cross sections, we use the integrated luminosities collected at the different centre-of-mass energies between $130 \mathrm{GeV}$ and $172 \mathrm{GeV}$ without assuming a scaling of the cross sections. Hence our limits correspond to luminosity weighted average cross sections.

Assuming a branching fraction for $\tilde{\ell}^{ \pm} \rightarrow \tilde{\chi}_{1}^{0} \ell^{ \pm}$of $100 \%$, upper limits are set on production cross sections for scalar electrons, muons and taus in the plane $M_{\tilde{\chi}_{1}^{0}}$ versus $M_{\tilde{\ell}^{ \pm}}$as depicted in Figure 3. The efficiency for the selection of scalar electrons includes the $t$-channel contribution. For scalar electron and muon masses below $80 \mathrm{GeV}$, and $\Delta M$ sufficiently large, cross sections above $0.55 \mathrm{pb}$ and $0.38 \mathrm{pb}$ are excluded, respectively. Owing to the lower selection efficiency and the presence of a candidate event, the corresponding upper limit for the scalar tau cross section is $1.3 \mathrm{pb}$. Exclusions in the mass range $80-86 \mathrm{GeV}$ are less stringent because only data taken at $\sqrt{s}=172 \mathrm{GeV}$ contribute.

The contours of upper limits on the production cross sections for the process $\mathrm{e}^{+} \mathrm{e}^{-} \rightarrow \tilde{\chi}_{1}^{ \pm} \tilde{\chi}_{1}^{\mp}$ are shown in Figure 4 assuming $\tilde{\chi}_{1}^{ \pm} \rightarrow \mathrm{W}^{*} \tilde{\chi}_{1}^{0}$ for the chargino decays with standard W branching fractions a) and for purely leptonic $\mathrm{W}^{*}$ decays $\mathrm{b}$ ). In parts of the kinematically accessible region, a cross section as low as $0.5 \mathrm{pb}$ is excluded for standard $\mathrm{W}$ branching fractions.

Similarly, cross section limits for associated neutralino production $\mathrm{e}^{+} \mathrm{e}^{-} \rightarrow \tilde{\chi}_{1}^{0} \tilde{\chi}_{2}^{0}$ are derived, as shown in Figure 5, assuming only $\mathrm{Z}^{*} \tilde{\chi}_{1}^{0}$ transitions in $\tilde{\chi}_{2}^{0}$ decays. For $M_{\tilde{\chi}_{2}^{0}}$ larger than $\approx 110 \mathrm{GeV}$ and $\Delta M>10 \mathrm{GeV}$ a cross section larger than $1.5 \mathrm{pb}$ is ruled out.

\subsection{Interpretation in the MSSM}

In the MSSM, with Grand Unification assumption [22], the masses and couplings of the gauginos and of the SUSY particles as well as their production cross sections, are entirely described [5] once five parameters are fixed: $\tan \beta$ (the ratio of the vacuum expectation values of the two Higgs doublets), $M \equiv M_{2}$ (the gaugino mass parameter), $\mu$ (the higgsino mixing parameter), $m_{0}$ (the common mass for scalar fermions at the GUT scale) and $A$ (the trilinear coupling in the Higgs sector). The assumption of a common scalar mass scale is relevant only if light fermion partners are considered. We investigate the following MSSM parameter space:

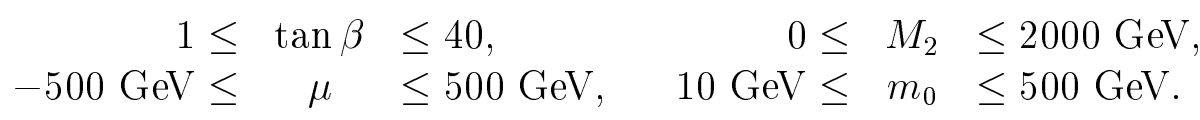

The interpretation of the search results in the MSSM presented here do not depend on the value of $A$.

All the limits on the cross sections previously shown can be translated into exclusion regions in the MSSM parameter space. To derive such limits, we use the generator SUSYGEN [16] where the cross sections and branching fractions are calculated in the MSSM framework.

\subsubsection{Limits on scalar lepton masses}

In general, the SUSY partners of the right-handed leptons $\left(\tilde{\ell}_{R}^{ \pm}\right)$are expected to be lighter than their counterparts for left-handed leptons. Hence, we show in Figure 6a) the exclusion contour in 
the $M_{\tilde{\chi}_{1}^{0}}-M_{\tilde{\mathrm{e}}_{R}^{ \pm}}$plane considering only the reaction $\mathrm{e}^{+} \mathrm{e}^{-} \rightarrow \widetilde{\mathrm{e}}_{R}^{ \pm} \tilde{\mathrm{e}}_{R}^{\mp}$ and setting $\mu=-200 \mathrm{GeV}$ and $\tan \beta=1.41$. To derive this contour, only the purely leptonic decay $\tilde{\mathrm{e}}_{R}^{ \pm} \rightarrow \mathrm{e}^{ \pm} \tilde{\chi}_{1}^{0}$ is considered, neglecting the efficiency for cascade decays. For $M_{\tilde{\chi}_{1}^{0}}<48.8 \mathrm{GeV}$ and $\Delta M \geq 5 \mathrm{GeV}$, we exclude a scalar electron lighter than $70 \mathrm{GeV}$ for $\tan \beta=1.41$. As the cross section increases with $\tan \beta$, this limit holds also for larger $\tan \beta$. This significantly improves our result obtained at $\sqrt{s}=130-140 \mathrm{GeV}[2]$.

With the same assumptions, and considering only the reaction $\mathrm{e}^{+} \mathrm{e}^{-} \rightarrow \tilde{\mu}_{R}^{ \pm} \tilde{\mu}_{R}^{\mp}$, we derive the lower limits on the scalar muon mass as a function of $M_{\tilde{\chi}_{1}^{0}}$ as shown in Figure $6 \mathrm{~b}$ ). In particular, for $M_{\tilde{\chi}_{1}^{0}}<50 \mathrm{GeV}$ and $\Delta M \geq 5 \mathrm{GeV}$, we exclude a scalar muon lighter than $60 \mathrm{GeV}$, independent of $\tan \beta$. This new limit improves the one obtained at the $\mathrm{Z}$ resonance [6].

Assuming universality for the scalar masses and combining the searches for charginos, neutralinos and scalar partners of the left- and right-handed electrons and muons, lower limits on the mass of the SUSY partners of the right-handed leptons as a function of $M_{\tilde{\chi}_{1}^{0}}$ are obtained, as shown in Figure 6c). A limit of $M_{\tilde{\ell}^{ \pm}} \geq 73.9 \mathrm{GeV}$ is reached for $M_{\tilde{\chi}_{1}^{0}}<47.7 \mathrm{GeV}$ and $\Delta M \geq$ $3 \mathrm{GeV}$. The exclusion of a light neutralino, described below, enables us to rule out a $\tilde{\ell}_{R}^{ \pm}$with a mass beyond the kinematic limit of $86 \mathrm{GeV}$.

\subsubsection{Limits on chargino and neutralino masses}

In the MSSM framework, while the cross sections and decay branching fractions of the charginos and neutralinos depend on the masses of the scalar leptons, their masses depend only on $M_{2}$, $\mu$ and $\tan \beta$. Therefore, exclusion regions can be expressed in the $M_{2}-\mu$ plane for a given $\tan \beta$. The exclusions in the high $m_{0}$ range are derived from chargino and neutralino searches, while for low $m_{0}$ the searches for scalar leptons also contribute. We also take into account all chargino and neutralino cascade decays:

- $\tilde{\chi}_{1}^{ \pm} \rightarrow \tilde{\chi}_{2}^{0} \mathrm{~W}^{*}$ : we observe a slight decrease of the efficiency relative to $\tilde{\chi}_{1}^{ \pm} \rightarrow \tilde{\chi}_{1}^{0} \mathrm{~W}^{*}$ depending on the masses of $\tilde{\chi}_{2}^{0}, \tilde{\chi}_{1}^{ \pm}$and $\tilde{\chi}_{1}^{0}$. Conservatively, the lowest efficiency is used for cascade decays.

- $\tilde{\chi}_{3,4}^{0} \rightarrow \tilde{\chi}_{2}^{0} Z^{*}$ : the efficiency is found to be larger than the efficiency achieved for the $\tilde{\chi}_{3,4}^{0} \rightarrow \tilde{\chi}_{1}^{0} \mathrm{Z}^{*}$ channel, especially in the large $\Delta M$ region. We use conservatively the efficiencies obtained in the latter, standard channel.

Substantial extension of the excluded regions with respect to the results obtained at $\sqrt{s}=$ $130-140 \mathrm{GeV}$ are reached, as shown in Figure 7 for $\tan \beta=1.41$ a) and $\tan \beta=40 \mathrm{~b}$ ). Our limits are extended by typically $20 \mathrm{GeV}$ on $\mu$ and $25 \mathrm{GeV}$ on $M_{2}$.

Depending on the neutralino-chargino field content, one distinguishes the following cases for the determination of lower limits on the neutralino and chargino masses:

- Higgsino-like $\tilde{\chi}_{2}^{0}$ and $\tilde{\chi}_{1}^{ \pm}\left(M_{2} \gg|\mu|\right)$ : in this case, the production cross sections do not depend on the scalar lepton masses, $\Delta M$ is low and decreases with increasing $M_{2}$. Consequently, the limits on the masses of the next to lightest neutralino and the lightest chargino decrease with $M_{2}$ as depicted in Figures 8a) and b), respectively. For $\tan \beta=$ 1.41 and $M_{2}$ less than $500 \mathrm{GeV}, M_{\tilde{\chi}_{2}^{0}} \leq 92 \mathrm{GeV}$ and $M_{\tilde{\chi}_{1}^{ \pm}} \leq 85 \mathrm{GeV}$ are excluded.

- Gaugino-like chargino $\left(|\mu| \gg M_{2}\right)$ : the cross section depends strongly on the scalar neutrino mass. For $50 \mathrm{GeV} \leq M_{\tilde{\nu}} \leq 80 \mathrm{GeV}$ the destructive interference term reduces the cross section by one order of magnitude compared to what is expected for $M_{\tilde{\nu}} \geq 500 \mathrm{GeV}$. 
When the two body decay $\tilde{\chi}_{1}^{ \pm} \rightarrow \ell^{ \pm} \tilde{\nu}$ is dominant, the relevant $\Delta M$ becomes $\Delta M=$ $M_{\tilde{\chi}_{1}^{ \pm}}-M_{\tilde{\nu}}$. In this case, for the low $\Delta M$ region, the acceptance for charginos decreases but we benefit there from the complementary scalar lepton searches, especially at low $\tan \beta$ values. The limits obtained in the $M_{\tilde{\chi}_{1}^{ \pm}}-M_{\tilde{\nu}}$ plane are shown in Figure 9 for $\tan \beta=1.41$ and two values of $\mu(-200 \mathrm{GeV}$ and $-500 \mathrm{GeV})$. The dashed line indicates the boundary $M_{\tilde{\chi}_{1}^{ \pm}}=M_{\tilde{\nu}}$. For small $M_{\tilde{\nu}}$ we can exclude chargino masses beyond the kinematic limit. For large $M_{\tilde{\nu}}$ and for $\mu=-500(200) \mathrm{GeV}$ we obtain $M_{\tilde{\chi}_{1}^{ \pm}}>85.5(84.0) \mathrm{GeV}$, which is close to the kinematic limit, independently of $\tan \beta$. Finally, for $\mu=-500(-200) \mathrm{GeV}$ a limit of $M_{\tilde{\chi}_{1}^{ \pm}}>79.8$ (69.2) $\mathrm{GeV}$ is obtained for any $M_{\tilde{\nu}}$ and for $\tan \beta \leq 1.41$.

We also derive limits on the mass of the lightest neutralino as a function of $m_{0}$ (Figure 10a) and as a function of $\tan \beta$ (Figure 10b). The results shown in Figure 10 are obtained from a scan in the MSSM parameter space in steps of $1 \mathrm{GeV}$ for $M_{2}, 0.5 \mathrm{GeV}$ for $\mu$ and $1 \mathrm{GeV}$ for $m_{0}$. The following values of $\tan \beta$ are used: 1.0, 1.2, 1.41, 1.7, 2.0, 2.2, 2.4, 3.0, 5.0, 10.0 and 40.0.

For low $m_{0}(\leq 65 \mathrm{GeV})$ the limit comes mainly from the scalar lepton searches. For large $m_{0}(\geq 200 \mathrm{GeV})$, where the limit is derived only from chargino and neutralino searches we obtain $M_{\tilde{\chi}_{1}^{0}}>24.6 \mathrm{GeV}$ for $\tan \beta \geq 1$. For small $\tan \beta$ values and for $\mu$ values in the range $-60 \mathrm{GeV}$ to $-70 \mathrm{GeV}$ the excluded domain still benefits from the results obtained with data at the $\mathrm{Z}$ peak.

In the intermediate region $\left(65 \mathrm{GeV} \leq m_{0} \leq 85 \mathrm{GeV}\right)$ the production cross section for charginos is minimal and the scalar neutrino is light enough to allow the following decay modes $\tilde{\chi}_{2,3,4}^{0} \rightarrow \tilde{\nu} \nu$ and $\tilde{\chi}_{1}^{ \pm} \rightarrow \tilde{\nu} l^{ \pm}$. The minimum allowed value for $M_{\tilde{\chi}_{1}^{0}}$ is found in the parameter space region where $\Delta M=M_{\tilde{\chi}_{1}^{ \pm}}-M_{\tilde{\nu}}$ is small and where the next to lightest neutralinos decay invisibly (i.e. for $m_{0}=67 \mathrm{GeV}, \mu=-104 \mathrm{GeV}, M_{2}=18 \mathrm{GeV}$ and $\tan \beta=2$ ). There, the exclusion contour is derived from the scalar lepton searches. If photonic final states are not taken into account, we find a deeper minimum for $M_{\tilde{\chi}_{1}^{0}}$ at lower $\tan \beta$ values (1.2-1.41). There, the next to lightest neutralino decays almost $100 \%$ into $\gamma \tilde{\chi}_{1}^{0}$ and searches for single and multi photons plus missing energy [23] become important, as can be seen in Figure 10b). Finally, we obtain a lower limit for the lightest neutralino of $M_{\tilde{\chi}_{1}^{0}} \geq 10.9 \mathrm{GeV}$ for $\tan \beta \geq 1$ and for any $m_{0}$ value.

\section{Conclusion}

No evidence for SUSY particles is found since 8 candidate events survive the cuts when a number of 7.6 background events is expected. This enables us, using also data at lower centre-of-mass energies, to set new upper limits on their production cross sections. In the MSSM framework, assuming also scalar lepton mass universality and gauge mass unification, the following $95 \%$ C.L. lower limits are derived:

- $M_{\tilde{\ell}^{ \pm}}>73.9 \mathrm{GeV}$ for $M_{\tilde{\chi}_{1}^{0}} \leq 47.7 \mathrm{GeV},|\mu| \geq 200 \mathrm{GeV}$ and $\tan \beta \geq 1.41$;

- $M_{\tilde{\chi}_{1}^{ \pm}}>85.5 \mathrm{GeV}$ for a Gaugino-like chargino with $\mathrm{M}_{\tilde{\nu}} \geq 300 \mathrm{GeV}$ and $\mu=-500 \mathrm{GeV}$; $M_{\tilde{\chi}_{1}^{ \pm}}>79.8 \mathrm{GeV}$ for any $\mathrm{M}_{\tilde{\nu}}, \tan \beta \leq 1.41$ and $\mu=-500 \mathrm{GeV}$;

- $M_{\tilde{\chi}_{1}^{ \pm}}>85.0 \mathrm{GeV}$ for a Higgsino-like chargino assuming $M_{2} \leq 500 \mathrm{GeV}$ and $\tan \beta=1.41$;

- $M_{\tilde{\chi}_{2}^{0}}>92.0 \mathrm{GeV}$ for a Higgsino-like neutralino assuming $M_{2} \leq 500 \mathrm{GeV}$ and $\tan \beta=1.41$;

- $M_{\tilde{\chi}_{1}^{0}}>24.6 \mathrm{GeV}$ for $m_{0} \geq 200 \mathrm{GeV}$ and $\tan \beta \geq 1 ; M_{\tilde{\chi}_{1}^{0}}>10.9 \mathrm{GeV}$ for any $m_{0}$ value.

These results improve significantly our previous limits. 


\section{Acknowledgements}

We wish to congratulate the CERN accelerator divisions for the successful upgrade of the LEP machine and to express our gratitude for the excellent performance of the machine. We acknowledge the effort of all the engineers, technicians and support staff who have participated in the construction and maintenance of this experiment.

\section{References}

[1] Y.A. Godfand and E.P. Likthman, JETP Lett.13 (1971);

D.V. Volkhov and V.P Akulov, Phys. Lett. B 46 (1973) 109;

J. Wess and B. Zumino, Nucl. Pys. B 70 (1974) 39;

P. Fayet and S. Ferrara, Phys. Rep. 32 (1977) 249;

A. Salam and J. Strathdee, Fortschr. Phys. 26 (1978) 57.

[2] L3 Collab., M. Acciari et al., Phys. Lett. B 377 (1996) 289.

[3] ALEPH Collab., D. Buskulic et al., Phys. Lett. B 373 (1996) 246; Z. Phys. C 72 (1996) 549; R. Barate et al., Phys. Lett. B 407 (1997) 377; R. Barate et al., Preprints CERNPPE/97-84, CERN-PPE/97-122, submitted to Phys. Lett.B;

DELPHI Collab., P. Abreu et al., Phys. Lett. B 387 (1996) 651; Phys. Lett. B 382 (1996) 323; Preprint CERN-PPE/97-107 submitted to Z. Phys. C;

OPAL Collaboration, K. Ackerstaff et al., Phys. Lett. B 377 (1996) 273, Phys. Lett. B 389 (1996) 616, Phys. Lett. B 396 (1997) 301, G. Alexander et al., Z. Phys. C 73 (1997) 201; K. Ackerstaff et al., Preprints CERN-PPE/97-83, CERN-PPE/97-124, submitted to Z. Phys. C.

[4] CDF Collab., F. Abe et al., Phys. Rev. Lett. 76 (1996) 4307;

D0 Collab., S. Abachi et al., Phys. Rev. Lett. 76 (1996) 2228.

[5] See for instance:

H.P. Nilles, Phys. Rep. 110 (1984)1;

H.E. Haber and G.L. Kane, Phys. Rep. 117 (1985) 75;

R. Barbieri, Riv. Nuovo Cim. $11 \mathrm{n}^{\circ} 4$ (1988) 1.

[6] L3 Collab., O. Adriani et al., Phys. Rep. 236 (1993) 1.

[7] L3 Collab., M. Acciarri et al., Phys. Lett. B 350 (1995) 109.

[8] L3 Collab., B. Adeva et al., Nucl. Instr. and Meth. A 289 (1990) 35;

M. Chemarin et al., Nucl. Instr. and Meth. A 349 (1994) 345; M. Acciari et al., Nucl. Instr. and Meth. A 351 (1994) 300; G. Basti et al., Nucl. Instr. and Meth. A 374 (1996) 293; I. Brock et al., Nucl. Instr. and Meth. A 381 (1996) 236; A. Adam et al., Nucl. Instr. and Meth. A 383 (1996) 342.

[9] T. Sjöstrand, PYTHIA 5.7 and JETSET 7.4 Physics and Manual, CERN-TH/7112/93 (1993), revised August 1995; Comp. Phys. Comm. 82 (1994) 74.

[10] F.A. Berends, R. Kleiss and R. Pittau, Nucl. Phys. B 424 (1994) 308; Nucl. Phys. B 426 (1994) 344; Nucl. Phys. (Proc. Suppl.) B 37 (1994) 163; Phys. Lett. B 335 (1994) 490; Comp. Phys. Comm. 83 (1994) 141. 
[11] KORALZ version 4.02 is used.

S. Jadach, B.F.L. Ward and Z. Wạs, Comp. Phys. Comm. 79 (1994) 503.

[12] BHAGENE version 3 is used.

J.H. Field, Phys. Lett. B 323 (1994) 432;

J.H. Field and T. Riemann, Comp. Phys. Comm. 94 (1996) 53.

[13] KORALW version 1.21 is used.

M. Skrzypek, S. Jadach, W. Placzek and Z. Wa̧s, Comp. Phys. Comm. 94 (1996) 216;

M. Skrzypek, S. Jadach, M. Martinez, W. Placzek and Z. Wạs, Phys. Lett. B 372 (1996) 289.

[14] F.A. Berends, P.H. Daverfeldt and R. Kleiss, Nucl. Phys. B 253 (1985) 441.

[15] PHOJET version 1.05 is used.

R. Engel, Z. Phys. C 66 (1995) 203;

R. Engel and J. Ranft, Phys. Rev. D 54 (1996) 4244.

[16] The SUSYGEN generator, S. Katsanavas and M. Melachroinos, Proceedings of the Workshop "Physics at LEP2", Report CERN 96-01 (1996), eds. G. Altarelli, T. Sjöstrand, F. Zwirner, Vol.2, p. 328.

[17] The DFGT generator, C. Dionisi et al., Proceedings of the Workshop "Physics at LEP2", Report CERN 96-01 (1996), eds. G. Altarelli, T. Sjöstrand, F. Zwirner, Vol.2, p. 337.

[18] The L3 detector simulation is based on GEANT Version 3.15. See R. Brun et al., "GEANT3", CERN DD/EE/84-1 (Revised), September 1987; the GHEISHA program (H. Fesefeldt, RWTH Aachen Report PITHA 85/02 (1985)) is used to simulate hadronic interactions.

[19] S. Catani et al., Phys. Lett. B 269 (1991) 432;

S. Bethke et al., Nucl. Phys. B370 (1992) 310.

[20] J.F Grivaz, F. Le Diberder, preprint LAL-92-37, June 1992.

[21] R.D. Cousins and V.L. Highland, Nucl. Instr. and Meth. A 320 (1992) 331.

[22] See for instance:

L. Ibanez, Phys. Lett. B 118 (1982) 73;

R. Barbieri, S. Farrara, C. Savoy, Phys. Lett. B 119 (1982) 343.

[23] L3 Collab., M. Acciarri et al., "Single and Multi-Photon Events with Missing Energy in $\mathrm{e}^{+} \mathrm{e}^{-}$Collisions at $161 \mathrm{GeV}<\sqrt{s}<172 \mathrm{GeV}$ ", Preprint CERN-PPE/97-76; accepted for publication in Phys. Lett B. 


\section{The L3 Collaboration:}

M.Acciarri ${ }^{29}$ O.Adriani, ${ }^{18}$ M.Aguilar-Benitez ${ }^{28}$ S.Ahlen, ${ }^{12}$ J.Alcaraz ${ }^{28}$ G.Alemanni ${ }^{24}$ J.Allaby, ${ }^{19}$ A.Aloisio, ${ }^{31}$ G.Alverson, ${ }^{13}$ M.G.Alviggi ${ }^{31}$ G.Ambrosi ${ }^{21}$ H.Anderhub, ${ }^{51}$ V.P.Andreev, ${ }^{7,40}$ T.Angelescu ${ }^{14}$ F.Anselmo, ${ }^{10}$ A.Arefiev ${ }^{30}$ T.Azemoon, T.Aziz, ${ }^{11}$ P.Bagnaia, ${ }^{39}$ L.Baksay, ${ }^{46}$ S.Banerjee, ${ }^{11}$ Sw.Banerjee, ${ }^{11}$ K.Banicz, ${ }^{48}$ A.Barczyk, ${ }^{51,49}$ R.Barillère ${ }^{19}$ L.Barone ${ }^{39}$ P.Bartalini ${ }^{36}$ A.Baschirotto ${ }^{29}$ M.Basile, ${ }^{10}$ R.Battiston ${ }^{36}$ A.Bay ${ }^{24}$ F.Becattini ${ }^{18}$ U.Becker, ${ }^{17}$ F.Behner ${ }^{51}$ J.Berdugo ${ }^{28}$ P.Berges, ${ }^{17}$ B.Bertucci ${ }^{36}$ B.L.Betev ${ }^{51}$ S.Bhattacharya ${ }^{11}$ M.Biasini ${ }^{19}$ A.Biland ${ }^{51}$ G.M.Bilei ${ }^{36}$ J.J.Blaising, ${ }^{4}$ S.C.Blyth ${ }^{37}$ G.J.Bobbink, R.Bock, ${ }^{1}$ A.Böhm, ${ }^{1}$ L.Boldizsar ${ }^{15}$ B.Borgia ${ }^{39}$ D.Bourilkov, ${ }^{51}$ M.Bourquin, ${ }^{21}$ S.Braccini, ${ }^{21}$ J.G.Branson, ${ }^{42}$ V.Brigljevic ${ }^{51}$ I.C.Brock, ${ }^{37}$ A.Buffini ${ }^{18}$ A.Buijs, ${ }^{47}$ J.D.Burger ${ }^{17}$ W.J.Burger, ${ }^{21}$ J.Busenitz, ${ }^{46}$ A.Button, ${ }^{3}$ X.D.Cai,${ }^{17}$ M.Campanelli, ${ }^{51}$ M.Capell, ${ }^{17}$ G.Cara Romeo, ${ }^{10}$ G.Carlino, ${ }^{31}$ A.M.Cartacci ${ }^{18}$ J.Casaus ${ }^{28}$ G.Castellini, ${ }^{18}$ F.Cavallari ${ }^{39}$ N.Cavallo, ${ }^{31}$ C.Cecchi ${ }^{21}$ M.Cerrada, ${ }^{28}$ F.Cesaroni, ${ }^{25}$ M.Chamizo, ${ }^{28}$ Y.H.Chang, ${ }^{53}$ U.K.Chaturvedi ${ }^{20}$ S.V.Chekanov ${ }^{33}$ M.Chemarin ${ }^{27}$ A.Chen ${ }^{53}$ G.Chen, ${ }^{8}$ G.M.Chen ${ }^{8}$ H.F.Chen ${ }^{22}$ H.S.Chen ${ }^{8}$ X.Chereau, G.Chiefari ${ }^{31}$ C.Y.Chien ${ }^{5}$ L.Cifarelli, ${ }^{41}$ F.Cindolo, ${ }^{10}$ C.Civinini, ${ }^{18}$ I.Clare ${ }^{17}$ R.Clare ${ }^{17}$ H.O.Cohn ${ }^{34}$ G.Coignet, ${ }^{4}$ A.P.Colijn ${ }^{2}$ N.Colino ${ }^{28}$ S.Costantini, ${ }^{9}$ F.Cotorobai, ${ }^{14}$ B.de la Cruz ${ }^{28}$ A.Csilling, ${ }^{15}$ T.S.Dai ${ }^{17}$ R.D'Alessandro, ${ }^{18}$ R.de Asmundis, ${ }^{31}$ A.Degré, ${ }^{4}$ K.Deiters ${ }^{49}$ D.della Volpe, ${ }^{31}$ P.Denes, ${ }^{38}$ F.DeNotaristefani ${ }^{39}$ D.DiBitonto, ${ }^{46}$ M.Diemoz ${ }^{39}$ D.van Dierendonck, ${ }^{2}$ F.Di Lodovico, ${ }^{51}$ C.Dionisi ${ }^{39}$ M.Dittmar ${ }^{51}$ A.Dominguez, ${ }^{42}$ A.Doria, ${ }^{31}$ M.T.Dova, ${ }^{20, \sharp}$ D.Duchesneau, ${ }^{4}$ P.Duinker, ${ }^{2}$ I.Duran ${ }^{43}$ S.Dutta, ${ }^{11}$ S.Easo, ${ }^{36}$

Yu.Efremenko, ${ }^{34}$ H.El Mamouni, ${ }^{27}$ A.Engler, ${ }^{37}$ F.J.Eppling, ${ }^{17}$ F.C.Erné, ${ }^{2}$ J.P.Ernenwein ${ }^{27}$ P.Extermann ${ }^{21}$ M.Fabre, ${ }^{49}$ R.Faccini ${ }^{39}$ S.Falciano, ${ }^{39}$ A.Favara ${ }^{18}$ J.Fay ${ }^{27}$ O.Fedin,${ }^{40}$ M.Felcini, ${ }^{51}$ B.Fenyi, ${ }^{46}$ T.Ferguson, ${ }^{37}$ F.Ferroni ${ }^{39}$

H.Fesefeldt ${ }^{1}$ E.Fiandrini, ${ }^{36}$ J.H.Field ${ }^{21}$ F.Filthaut ${ }^{37}$ P.H.Fisher ${ }^{17}$ I.Fisk ${ }^{42}$ G.Forconi ${ }^{17}$ L.Fredj ${ }^{21}$ K.Freudenreich ${ }^{51}$ C.Furetta ${ }^{29}$ Yu.Galaktionov, ${ }^{30,17}$ S.N.Ganguli ${ }^{11}$ P.Garcia-Abia ${ }^{50}$ S.S.Gau, ${ }^{13}$ S.Gentile, ${ }^{39}$ N.Gheordanescu, ${ }^{14}$ S.Giagu, ${ }^{39}$ S.Goldfarb, ${ }^{24}$ J.Goldstein, ${ }^{12}$ Z.F.Gong, ${ }^{22}$ A.Gougas, G.Gratta, ${ }^{35}$ M.W.Gruenewald, ${ }^{5}$ V.K.Gupta, ${ }^{38}$ A.Gurtu ${ }^{11}$ L.J.Gutay, ${ }^{48}$ B.Hartmann, ${ }^{1}$ A.Hasan, ${ }^{32}$ D.Hatzifotiadou, ${ }^{10}$ T.Hebbeker, A.Hervé, ${ }^{19}$ W.C.van Hoek ${ }^{33}$ H.Hofer ${ }^{11}$ S.J.Hong, ${ }^{45}$ H.Hoorani, ${ }^{37}$ S.R.Hou, ${ }^{53}$ G.Hu, ${ }^{5}$ V.Innocente, ${ }^{19}$ K.Jenkes, B.N.Jin, L.W.Jones, ${ }^{3}$ P.de Jong, ${ }^{19}$ I.Josa-Mutuberria, ${ }^{28}$ A.Kasser, ${ }^{24}$ R.A.Khan ${ }^{20}{ }^{\text {D.Kamrad }}{ }^{50}$ Yu.Kamyshkov, ${ }^{34}$ J.S.Kapustinsky ${ }^{26}$ Y.Karyotakis, M.Kaur ${ }^{20, \diamond}$ M.N.Kienzle-Focacci ${ }^{21}$ D.Kim ${ }^{39}$ D.H.Kim ${ }^{45}$ J.K.Kim ${ }^{45}$ S.C.Kim, ${ }^{45}$ Y.G.Kim,${ }^{45}$ W.W.Kinnison, ${ }^{26}$ A.Kirkby, ${ }^{35}$ D.Kirkby ${ }^{35}$ J.Kirkby ${ }^{19}$ D.Kiss, ${ }^{15}$ W.Kittel ${ }^{33}$ A.Klimentov, ${ }^{17,30}$ A.C.König, ${ }^{33}$ A.Kopp, ${ }^{50}$ I.Korolko, $^{30}$ V.Koutsenko, ${ }^{17,30}$ R.W.Kraemer, ${ }^{37}$ W.Krenz, ${ }^{1}$ A.Kunin, ${ }^{17,30}$ P.Lacentre, ${ }^{50,4, \sharp}$ P.Ladron de Guevara, ${ }^{28}$ I.Laktineh $^{27}$ G.Landi ${ }^{18}$ C.Lapoint ${ }^{17}$ K.Lassila-Perini ${ }^{51}$ P.Laurikainen, ${ }^{23}$ M.Lebeau ${ }^{19}$ A.Lebedev ${ }^{17}$ P.Lebrun ${ }^{27}$ P.Lecomte ${ }^{51}$ P.Lecoq ${ }^{19}$ P.Le Coultre ${ }^{51}$ H.J.Lee ${ }^{9}$ J.M.Le Goff ${ }^{19}$ R.Leiste ${ }^{50}$ E.Leonardi, ${ }^{39}$ P.Levtchenko, ${ }^{40}$ C.Li ${ }^{22}$ C.H.Lin ${ }^{53}$ W.T.Lin ${ }^{53}$ F.L.Linde, ${ }^{2,19}$ L.Lista $^{31}$ Z.A.Liu, ${ }^{8}$ W.Lohmann ${ }^{50}$ E.Longo, ${ }^{39}$ W.Lu ${ }^{35}$ Y.S.Lu, ${ }^{8}$ K.Lübelsmeyer, ${ }^{1}$ C.Luci ${ }^{39}$ D.Luckey ${ }^{17}$ L.Luminari ${ }^{39}$ W.Lustermann ${ }^{49}$ W.G.Ma ${ }^{22}$ M.Maity ${ }^{11}$ G.Majumder, ${ }^{11}$ L.Malgeri ${ }^{39}$ A.Malinin ${ }^{30}$ C.Maña ${ }^{28}$ D.Mangeol ${ }^{33}$ S.Mangla ${ }^{11}$ P.Marchesini, ${ }^{51}$ A.Marin ${ }^{12}$ J.P.Martin ${ }^{27}$ F.Marzano, ${ }^{39}$ G.G.G.Massaro, D.McNally, ${ }^{19}$ R.R.McNeil, ${ }^{7}$ S.Mele, ${ }^{31}$ L.Merola, ${ }^{31}$ M.Meschini, ${ }^{18}$ W.J.Metzger, ${ }^{33}$ M.von der Mey, ${ }^{1}$ Y.Mi, ${ }^{24}$ A.Mihul, ${ }^{14}$ A.J.W.van Mil ${ }^{33}$ H.Milcent, ${ }^{19}$ G.Mirabelli, ${ }^{39}$ J.Mnich $^{19}$ P.Molnar, ${ }^{9}$ B.Monteleoni, ${ }^{18}$ R.Moore, ${ }^{3}$ S.Morganti ${ }^{39}$ T.Moulik, ${ }^{11}$ R.Mount ${ }^{35}$ F.Muheim, ${ }^{21}$ A.J.M.Muijs ${ }^{2}$ S.Nahn,${ }^{17}$ M.Napolitano, ${ }^{31}$ F.Nessi-Tedaldi ${ }^{51}$ H.Newman ${ }^{35}$ T.Niessen ${ }^{1}$ A.Nippe, ${ }^{24}$ A.Nisati ${ }^{39}$ H.Nowak ${ }^{50}$ Y.D.Oh ${ }^{45}$ H.Opitz, G.Organtini ${ }^{39}$ R.Ostonen ${ }^{23}$ C.Palomares $^{28}$ D.Pandoulas, ${ }^{1}$ S.Paoletti, ${ }^{39}$ P.Paolucci ${ }^{31}$ H.K.Park ${ }^{37}$ I.H.Park ${ }^{45}$ G.Pascale ${ }^{39}$ G.Passaleva ${ }^{19}$ S.Patricelli ${ }^{31}{ }^{\text {T.Paul }}{ }^{13}$ M.Pauluzzi ${ }^{36}$ C.Paus ${ }^{19}$ F.Pauss ${ }^{51}$ D.Peach ${ }^{19}$ Y.J.Pei, ${ }^{1}$ S.Pensotti ${ }^{29}$ D.Perret-Gallix, ${ }^{4}$ B.Petersen ${ }^{33}$ S.Petrak, A.Pevsner, ${ }^{5}$ D.Piccolo, ${ }^{31}$ M.Pieri, ${ }^{18}$ P.A.Piroué ${ }^{38}{ }^{2}$ E.Pistolesi ${ }^{29}{ }^{2}$ V.Plyaskin, ${ }^{30}$ M.Pohl ${ }^{51}$ V.Pojidaev, ${ }^{30,18}$ H.Postema ${ }^{17}$ N.Produit ${ }^{21}$ D.Prokofiev, ${ }^{40}$ G.Rahal-Callot ${ }^{51}$ N.Raja ${ }^{11}$ P.G.Rancoita ${ }^{29}$ M.Rattaggi ${ }^{29}$ G.Raven,${ }^{42}$ P.Razis, ${ }^{32}$ K.Read ${ }^{34}$ D.Ren,${ }^{51}$ M.Rescigno, ${ }^{39}$ S.Reucroft, ${ }^{13}$ T.van Rhee, ${ }^{47}$ S.Riemann, ${ }^{50}$ K.Riles, ${ }^{3}$ A.Robohm, ${ }^{51}$ J.Rodin, ${ }^{17}$ B.P.Roe, L.Romero, ${ }^{28}$ S.Rosier-Lees, ${ }^{4}$ Ph.Rosselet, ${ }^{24}$ W.van Rossum, ${ }^{47}$ S.Roth, ${ }^{1}$ J.A.Rubio, ${ }^{19}$ D.Ruschmeier ${ }^{9}$ H.Rykaczewski, ${ }^{51}$ J.Salicio, ${ }^{19}$ E.Sanchez, ${ }^{28}$ M.P.Sanders ${ }^{33}$ M.E.Sarakinos ${ }^{23}{ }^{2}$ S.Sarkar, ${ }^{11}$ C.Schäfer ${ }^{1}$ V.Schegelsky, ${ }^{40}$ S.Schmidt-Kaerst, D.Schmitz, N.Scholz, ${ }^{1}$ H.Schopper, ${ }^{12}$ D.J.Schotanus, ${ }^{13}$ J.Schwenke, G.Schwering, C.Sciacca, ${ }^{31}$ D.Sciarrino, ${ }^{21}$ L.Servoli ${ }^{36}{ }^{1}$ S.Shevchenko, ${ }^{35}$ N.Shivarov, ${ }^{44}$ V.Shoutko, ${ }^{30}$ J.Shukla, ${ }^{26}$ E.Shumilov, ${ }^{30}$ A.Shvorob, ${ }^{35}$ T.Siedenburg, ${ }^{1}$ D.Son ${ }^{45}$ B.Smith ${ }^{17}$ P.Spillantini ${ }^{18}$ M.Steuer ${ }^{17}$ D.P.Stickland ${ }^{38}$ A.Stone, ${ }^{7}$ H.Stone, ${ }^{38}$ B.Stoyanov ${ }^{44}$ A.Straessner, ${ }^{1}$ K.Strauch ${ }^{16}$ K.Sudhakar, ${ }^{11}$ G.Sultanov, ${ }^{20}$ L.Z.Sun ${ }^{22}$ G.F.Susinno, ${ }^{21}$ H.Suter, ${ }^{51}$ J.D.Swain ${ }^{20}$ X.W.Tang, ${ }^{8}$ L.Tauscher, ${ }^{6}$ L.Taylor ${ }^{13}$ Samuel C.C.Ting, ${ }^{17}$ S.M.Ting, ${ }^{17}$ S.C.Tonwar, ${ }^{11}$ J.Tóth ${ }^{15}$ C.Tully, ${ }^{38}$ H.Tuchscherer, ${ }^{46}$ K.L.Tung ${ }^{8}{ }^{5}$ Y.Uchida ${ }^{17}$ J.Ulbricht ${ }^{51}$ U.Uwer, ${ }^{19}$ E.Valente ${ }^{39}$ R.T.Van de Walle ${ }^{33}$ G.Vesztergombi, ${ }^{15}$ I.Vetlitsky, ${ }^{30}$ G.Viertel ${ }^{51}$ M.Vivargent, ${ }^{4}$ R.Völkert, ${ }^{50}$ H.Vogel ${ }^{37}$ H.Vogt ${ }^{50}$ I.Vorobiev, ${ }^{19,30}$ A.A.Vorobyov, ${ }^{40}$ A.Vorvolakos, ${ }^{32}$ M.Wadhwa, ${ }^{6}$ W.Wallraff, ${ }^{1}$ J.C.Wang, ${ }^{17}$ X.L.Wang, ${ }^{22}$ Z.M.Wang, ${ }^{22}$ A.Weber, ${ }^{1}$ S.X.Wu, ${ }^{20}$ S.Wynhoff, ${ }^{1} . X u{ }^{12}$ Z.Z.Xu, ${ }^{22}$ B.Z.Yang, ${ }^{22}$ C.G.Yang, X.Y.Yao, ${ }^{8}$ J.B.Ye, ${ }^{22}$ S.C.Yeh, ${ }^{53}$ J.M.You, ${ }^{37}$ An.Zalite, ${ }^{40}$ Yu.Zalite, ${ }^{40}$ P.Zemp ${ }^{51}$ Y.Zeng, ${ }^{1}$ Z.Zhang, ${ }^{8}$ Z.P.Zhang, ${ }^{22}$ B.Zhou, ${ }^{12}$ G.Y.Zhu, R.Y.Zhu, ${ }^{35}$ A.Zichichi, ${ }^{10,19,20}$ F.Ziegler. ${ }^{50}$ 
1 I. Physikalisches Institut, RWTH, D-52056 Aachen, FRG ${ }^{\S}$

III. Physikalisches Institut, RWTH, D-52056 Aachen, FRG ${ }^{\S}$

2 National Institute for High Energy Physics, NIKHEF, and University of Amsterdam, NL-1009 DB Amsterdam, The Netherlands

3 University of Michigan, Ann Arbor, MI 48109, USA

4 Laboratoire d'Annecy-le-Vieux de Physique des Particules, LAPP,IN2P3-CNRS, BP 110, F-74941 Annecy-le-Vieux CEDEX, France

5 Johns Hopkins University, Baltimore, MD 21218, USA

6 Institute of Physics, University of Basel, CH-4056 Basel, Switzerland

7 Louisiana State University, Baton Rouge, LA 70803, USA

8 Institute of High Energy Physics, IHEP, 100039 Beijing, China ${ }^{\triangle}$

9 Humboldt University, D-10099 Berlin, FRG $^{\S}$

10 University of Bologna and INFN-Sezione di Bologna, I-40126 Bologna, Italy

11 Tata Institute of Fundamental Research, Bombay 400 005, India

12 Boston University, Boston, MA 02215, USA

13 Northeastern University, Boston, MA 02115, USA

14 Institute of Atomic Physics and University of Bucharest, R-76900 Bucharest, Romania

15 Central Research Institute for Physics of the Hungarian Academy of Sciences, H-1525 Budapest 114, Hungary ${ }^{\ddagger}$

16 Harvard University, Cambridge, MA 02139, USA

17 Massachusetts Institute of Technology, Cambridge, MA 02139, USA

18 INFN Sezione di Firenze and University of Florence, I-50125 Florence, Italy

19 European Laboratory for Particle Physics, CERN, CH-1211 Geneva 23, Switzerland

20 World Laboratory, FBLJA Project, CH-1211 Geneva 23, Switzerland

21 University of Geneva, CH-1211 Geneva 4, Switzerland

22 Chinese University of Science and Technology, USTC, Hefei, Anhui 230 029, China ${ }^{\triangle}$

23 SEFT, Research Institute for High Energy Physics, P.O. Box 9, SF-00014 Helsinki, Finland

24 University of Lausanne, CH-1015 Lausanne, Switzerland

25 INFN-Sezione di Lecce and Universitá Degli Studi di Lecce, I-73100 Lecce, Italy

26 Los Alamos National Laboratory, Los Alamos, NM 87544, USA

27 Institut de Physique Nucléaire de Lyon, IN2P3-CNRS,Université Claude Bernard, F-69622 Villeurbanne, France

28 Centro de Investigaciones Energeticas, Medioambientales y Tecnologicas, CIEMAT, E-28040 Madrid, Spainb

29 INFN-Sezione di Milano, I-20133 Milan, Italy

30 Institute of Theoretical and Experimental Physics, ITEP, Moscow, Russia

31 INFN-Sezione di Napoli and University of Naples, I-80125 Naples, Italy

32 Department of Natural Sciences, University of Cyprus, Nicosia, Cyprus

33 University of Nijmegen and NIKHEF, NL-6525 ED Nijmegen, The Netherlands

34 Oak Ridge National Laboratory, Oak Ridge, TN 37831, USA

35 California Institute of Technology, Pasadena, CA 91125, USA

36 INFN-Sezione di Perugia and Universitá Degli Studi di Perugia, I-06100 Perugia, Italy

37 Carnegie Mellon University, Pittsburgh, PA 15213, USA

38 Princeton University, Princeton, NJ 08544, USA

39 INFN-Sezione di Roma and University of Rome, "La Sapienza", I-00185 Rome, Italy

40 Nuclear Physics Institute, St. Petersburg, Russia

41 University and INFN, Salerno, I-84100 Salerno, Italy

42 University of California, San Diego, CA 92093, USA

43 Dept. de Fisica de Particulas Elementales, Univ. de Santiago, E-15706 Santiago de Compostela, Spain

44 Bulgarian Academy of Sciences, Central Lab. of Mechatronics and Instrumentation, BU-1113 Sofia, Bulgaria

45 Center for High Energy Physics, Korea Adv. Inst. of Sciences and Technology, 305-701 Taejon, Republic of Korea

46 University of Alabama, Tuscaloosa, AL 35486, USA

47 Utrecht University and NIKHEF, NL-3584 CB Utrecht, The Netherlands

48 Purdue University, West Lafayette, IN 47907, USA

49 Paul Scherrer Institut, PSI, CH-5232 Villigen, Switzerland

50 DESY-Institut für Hochenergiephysik, D-15738 Zeuthen, FRG

51 Eidgenössische Technische Hochschule, ETH Zürich, CH-8093 Zürich, Switzerland

52 University of Hamburg, D-22761 Hamburg, FRG

53 High Energy Physics Group, Taiwan, China

$\S$ Supported by the German Bundesministerium für Bildung, Wissenschaft, Forschung und Technologie

$\ddagger$ Supported by the Hungarian OTKA fund under contract numbers T14459 and T24011.

b Supported also by the Comisión Interministerial de Ciencia y Technología

\# Also supported by CONICET and Universidad Nacional de La Plata, CC 67, 1900 La Plata, Argentina

Ł Supported by Deutscher Akademischer Austauschdienst.

$\diamond$ Also supported by Panjab University, Chandigarh-160014, India 
$\triangle$ Supported by the National Natural Science Foundation of China. 


\begin{tabular}{|c|c|c|c|c|}
\hline \multicolumn{5}{|c|}{ Scalar electron selection } \\
\hline & & Low $\Delta M$ & Medium $\Delta M$ & Large $\Delta M$ \\
\hline$E_{l e p}(\mathrm{GeV})$ & $\leq$ & 23.7 & 43.6 & 67 \\
\hline$E_{l e p}(\mathrm{GeV})$ & $\geq$ & 2.63 & 19.7 & 14 \\
\hline$E_{l e p} / E_{v i s}$ & $\leq$ & 0.34 & 0.39 & 0.7 \\
\hline$p_{\perp}(\mathrm{GeV})$ & $\geq$ & 2.1 & 5.4 & 11 \\
\hline Acollinearity (rad) & $\leq$ & 2.0 & 2.6 & 3.13 \\
\hline Acoplanarity (rad) & $\leq$ & 3.1 & 3.0 & 3.0 \\
\hline$E_{25}^{\perp}(\mathrm{GeV})$ & $\leq$ & - & 1.8 & 4.4 \\
\hline$E_{25}(\mathrm{GeV})$ & $\leq$ & - & 4.5 & 2.2 \\
\hline$E_{60}^{b}(\mathrm{GeV})$ & $\leq$ & 5.5 & 5.4 & 3.1 \\
\hline $\sin \left(\theta_{\text {miss }}\right)$ & $\geq$ & 0.3 & 0.3 & 0.39 \\
\hline$E_{T T L}(\mathrm{GeV})$ & $\geq$ & 2.5 & 4.9 & 4.9 \\
\hline \multicolumn{5}{|c|}{ Scalar muon selection } \\
\hline$E_{l e p}(\mathrm{GeV})$ & $\leq$ & 24 & 39 & 73 \\
\hline$E_{l e p}(\mathrm{GeV})$ & $\geq$ & 2.1 & 19.7 & 10.6 \\
\hline$E_{l e p} / E_{v i s}$ & $\leq$ & 0.34 & 0.48 & 0.7 \\
\hline$p_{\perp}(\mathrm{GeV})$ & $\geq$ & 2.1 & 5.4 & 11 \\
\hline Acollinearity (rad) & $\leq$ & 3.1 & 3.0 & 3.1 \\
\hline Acoplanarity (rad) & $\leq$ & 3.1 & 3.0 & 3.0 \\
\hline$E_{25}^{\perp}(\mathrm{GeV})$ & $\leq$ & - & 6.3 & 6.5 \\
\hline$E_{25}(\mathrm{GeV})$ & $\leq$ & 0.7 & 7.2 & 1.6 \\
\hline$E_{60}^{b}(\mathrm{GeV})$ & $\leq$ & 9.6 & 3.3 & 4.9 \\
\hline $\sin \left(\theta_{\text {miss }}\right)$ & $\geq$ & 0.3 & 0.3 & 0.3 \\
\hline$E_{T T L}(\mathrm{GeV})$ & $\geq$ & 1.1 & 2.1 & 3.6 \\
\hline \multicolumn{5}{|c|}{ Scalar tau selection } \\
\hline$E_{l e p}(\mathrm{GeV})$ & $\leq$ & 19 & 26 & 29 \\
\hline$E_{l e p}(\mathrm{GeV})$ & $\geq$ & 2.1 & 10.6 & 15. \\
\hline$E_{\text {lep }} / E_{\text {vis }}$ & $\leq$ & 0.19 & 0.36 & 0.41 \\
\hline$p_{\perp}(\mathrm{GeV})$ & $\geq$ & 2.9 & 5.3 & 10.7 \\
\hline Acollinearity (rad) & $\leq$ & 3.1 & 2.7 & 2.5 \\
\hline Acoplanarity (rad) & $\leq$ & 3.0 & 3.0 & 2.5 \\
\hline$E_{25}^{\perp}(\mathrm{GeV})$ & $\leq$ & - & 1.7 & 1.8 \\
\hline$\theta_{123}^{l}(\mathrm{rad})$ & $\leq$ & - & 5.1 & 4.3 \\
\hline$E_{60}^{b}(\mathrm{GeV})$ & $\leq$ & - & 2.9 & 3.3 \\
\hline $\sin \left(\theta_{\text {miss }}\right)$ & $\geq$ & 0.66 & 0.6 & - \\
\hline$E_{T T L}(\mathrm{GeV})$ & $\geq$ & 2.1 & 4.8 & 2.5 \\
\hline
\end{tabular}

Table 1: Values of the cuts optimized for the scalar lepton searches and for various $\Delta \mathrm{M}$ ranges. They are determined with the optimization procedure described in section 3.1. 


\begin{tabular}{|l|c|c|c|c|}
\hline \multicolumn{5}{|c|}{ Inclusive lepton plus hadron selections } \\
\hline & & Low $\Delta M$ & Medium $\Delta M$ & Large $\Delta M$ \\
\hline No. of isolated lepton & $\geq$ & 1 & 1 & 1 \\
\hline$N_{t k}-N_{\text {lep }}$ & $\geq$ & 2 & 4 & 4 \\
\hline$N_{c l}$ & $\geq$ & 6 & 10 & 10 \\
\hline $\sin \left(\theta_{\text {miss }}\right)$ & $\geq$ & 0.55 & 0.38 & 0.36 \\
\hline$E_{25}^{\perp}(\mathrm{GeV})$ & $\leq$ & 6.9 & - & 7.9 \\
\hline$p_{\perp}(\mathrm{GeV})$ & $\geq$ & 5.5 & 3.2 & 4.7 \\
\hline$E_{\text {lep }}(\mathrm{GeV})$ & $\geq$ & 1.8 & 4.2 & 7.5 \\
\hline$E_{\text {lep }}(\mathrm{GeV})$ & $\leq$ & 12.4 & 42.6 & 38 \\
\hline$E_{T T J L}(\mathrm{GeV})$ & $\geq$ & 0.8 & - & - \\
\hline Acoplanarity $(\mathrm{rad})$ & $\leq$ & - & 2.97 & 3.04 \\
\hline$M_{\text {had }}(\mathrm{GeV})$ & $\leq$ & - & 34 & 46.3 \\
\hline$M_{\text {rec }}(\mathrm{GeV})$ & $\geq$ & 137 & 81.5 & 56 \\
\hline$E_{\text {vis }}(\mathrm{GeV})$ & $\geq$ & 4 & 5 & 5 \\
\hline$E_{\text {vis }}(\mathrm{GeV})$ & $\leq$ & 23.7 & 114 & 90 \\
\hline
\end{tabular}

Table 2: Values of the cuts for the lepton plus hadron selections; they are determined with the optimization procedure described in section 3.1.

\begin{tabular}{|l|c|c|c|c|}
\hline \multicolumn{5}{|c|}{ Purely hadronic selections } \\
\hline & & Low $\Delta M$ & Medium $\Delta M$ & Large $\Delta M$ \\
\hline$N_{c l}$ & $\geq$ & 11 & 11 & 11 \\
\hline$N_{t k}$ & $\geq$ & 5 & 5 & 5 \\
\hline$p_{\perp}(\mathrm{GeV})$ & $\geq$ & 6.8 & 7.9 & 23.7 \\
\hline$p_{\perp} / E_{\text {vis }}$ & $\geq$ & - & 0.24 & 0.29 \\
\hline$E_{\text {vis }}(\mathrm{GeV})$ & $\leq$ & 29.3 & 63.6 & 96.6 \\
\hline Acollinearity $(\mathrm{rad})$ & $\leq$ & 2.7 & 2.7 & 2.0 \\
\hline Acoplanarity $(\mathrm{rad})$ & $\leq$ & 3.1 & 2.5 & 2.9 \\
\hline $\sin \left(\theta_{\text {miss }}\right)$ & $\geq$ & 0.46 & 0.2 & 0.59 \\
\hline$E_{25}^{\perp}(\mathrm{GeV})$ & $\leq$ & 0.5 & 9.2 & 7.44 \\
\hline$E_{25}(\mathrm{GeV})$ & $\leq$ & - & - & 4.82 \\
\hline$p_{\|}$ & $\leq$ & 0.65 & 0.70 & 0.53 \\
\hline$M_{\text {vis }}(\mathrm{GeV})$ & $\geq$ & - & 15.7 & - \\
\hline$M_{\text {rec }}(\mathrm{GeV})$ & $\geq$ & - & 103 & 36.5 \\
\hline$E_{\text {vis }} / \sqrt{s}$ & $\geq$ & - & - & 0.36 \\
\hline$E_{T T J} / p_{\perp}$ & $\geq$ & 0.24 & - & - \\
\hline$y^{\perp}$ & $\geq$ & - & 0.23 & 0.28 \\
\hline
\end{tabular}

Table 3: Values of the cuts for the purely hadronic selections which are determined with the optimization procedure described in section 3.1 . 


\begin{tabular}{|c|c|c|c|c|c|c|}
\hline & \multicolumn{6}{|c|}{$\sqrt{s}=161 \mathrm{GeV}$} \\
\hline & \multirow{2}{*}{\multicolumn{2}{|c|}{$\begin{array}{c}M_{\tilde{\mathrm{e}}^{ \pm}}=70 \mathrm{GeV} \\
\tilde{\mathrm{e}}^{ \pm} \tilde{\mathrm{e}}^{\mp}\end{array}$}} & \multirow{2}{*}{\multicolumn{2}{|c|}{$\frac{M_{\tilde{\mu}^{ \pm}}=60 \mathrm{GeV}}{\tilde{\mu}^{ \pm} \tilde{\mu}^{\mp}}$}} & \multirow{2}{*}{\multicolumn{2}{|c|}{$\frac{M_{\tilde{\tau}^{ \pm}}=50 \mathrm{GeV}}{\tilde{\tau}^{ \pm} \tilde{\tau}^{\mp}}$}} \\
\hline & & & & & & \\
\hline$\Delta M(\mathrm{GeV})$ & $\epsilon(\%)$ & $N_{\exp }$ & $\epsilon(\%)$ & $N_{e x p}$ & $\epsilon(\%)$ & $N_{e x p}$ \\
\hline 5 & 31 & $<0.1$ & 24 & 0.2 & 3.1 & 0.3 \\
\hline 10 & 51 & 0.1 & 40 & 0.1 & 12 & 0.4 \\
\hline 20 & 60 & 0.1 & 41 & 0.1 & 17 & 0.5 \\
\hline 30 & 62 & 0.4 & 47 & 0.3 & 15 & 0.5 \\
\hline 40 & 61 & 0.4 & 46 & 0.3 & 15 & 0.5 \\
\hline 45 & 61 & 0.4 & 49 & 0.3 & 15 & 0.3 \\
\hline 55 & 58 & 0.4 & 45 & 0.3 & - & - \\
\hline \multirow[t]{4}{*}{65} & 47 & 0.4 & - & - & - & - \\
\hline & \multicolumn{6}{|c|}{$\sqrt{\bar{s}}=172 \mathrm{GeV}$} \\
\hline & \multirow{2}{*}{\multicolumn{2}{|c|}{$\begin{array}{c}M_{\tilde{\mathrm{e}}^{ \pm}}=75 \mathrm{GeV} \\
\tilde{\mathrm{e}}^{ \pm} \tilde{\mathrm{e}}^{\mp}\end{array}$}} & \multirow{2}{*}{\multicolumn{2}{|c|}{$\begin{array}{c}M_{\tilde{\mu}^{ \pm}}=65 \mathrm{GeV} \\
\tilde{\mu}^{ \pm} \tilde{\mu}^{\mp}\end{array}$}} & \multirow{2}{*}{\multicolumn{2}{|c|}{$\begin{array}{c}M_{\tilde{\tau}^{ \pm}}=55 \mathrm{GeV} \\
\tilde{\tau}^{ \pm} \tilde{\tau}^{\mp}\end{array}$}} \\
\hline & & & & & & \\
\hline$\Delta M(\mathrm{GeV})$ & $\epsilon(\%)$ & $N_{e x p}$ & $\epsilon(\%)$ & $N_{e x p}$ & $\epsilon(\%)$ & $N_{e x p}$ \\
\hline 5 & 30 & $<0.1$ & 21 & 0.1 & 2.9 & 0.3 \\
\hline 10 & 50 & 0.2 & 41 & 0.2 & 11 & 0.4 \\
\hline 20 & 60 & 0.2 & 39 & 0.2 & 18 & 0.7 \\
\hline 30 & 53 & 0.1 & 50 & 0.7 & 18 & 0.5 \\
\hline 40 & 61 & 0.7 & 45 & 0.7 & 18 & 0.5 \\
\hline 50 & 56 & 0.7 & 46 & 0.7 & 16 & 0.5 \\
\hline 60 & 59 & 0.7 & 44 & 0.7 & - & - \\
\hline 70 & 50 & 0.7 & - & - & - & - \\
\hline
\end{tabular}

Table 4: Scalar electron, scalar muon and scalar tau efficiencies $(\epsilon)$ and the number of events expected from Standard Model processes $\left(N_{\text {exp }}\right)$. Results are obtained at $\sqrt{s}=161 \mathrm{GeV}$ and $\sqrt{s}=172 \mathrm{GeV}$ as a function of $\Delta M$ for different $M_{\tilde{\ell}^{ \pm}}$values. 


\begin{tabular}{|c|c|c|c|c|c|c|c|c|}
\hline \multicolumn{3}{|c|}{$M_{\tilde{\chi}_{1}^{ \pm}}=80 \mathrm{GeV}$} & \multicolumn{6}{|c|}{$\sqrt{s}=161 \mathrm{GeV}$} \\
\hline & \multicolumn{2}{|c|}{$\overline{\overline{\mathrm{LH}}}$} & \multicolumn{2}{|c|}{$\overline{\mathrm{LL}}$} & \multicolumn{2}{|c|}{$\overline{\mathrm{HH}}$} & \multicolumn{2}{|c|}{$\overline{\overline{\chi_{\bar{\chi}}^{0}}}$} \\
\hline$\Delta M(\mathrm{GeV})$ & $\epsilon(\%)$ & $N_{e x p}$ & $\epsilon(\%)$ & $N_{e x p}$ & $\epsilon(\%)$ & $N_{\text {exp }}$ & $\epsilon(\%)$ & $N_{e x p}$ \\
\hline 5 & 4.2 & 0.5 & 1.7 & 0.3 & 5.5 & 0.2 & 4.3 & 0.6 \\
\hline 10 & 44 & 0.3 & 34 & 0.3 & 40 & 0.4 & 30 & 0.3 \\
\hline 20 & 58 & 0.1 & 48 & 0.2 & 64 & 0.2 & 50 & 0.3 \\
\hline 30 & 57 & 0.1 & 53 & 0.2 & 48 & 0.5 & 45 & 0.6 \\
\hline 40 & 68 & 0.3 & 50 & 0.1 & 39 & 0.6 & 44 & 0.8 \\
\hline 60 & 54 & 0.5 & 40 & 0.3 & 16 & 0.1 & 28 & 0.8 \\
\hline 75 & 17 & 0.6 & 20 & 0.3 & 3.2 & 0.1 & 9.3 & 0.9 \\
\hline \multicolumn{9}{|c|}{$M_{\tilde{\chi}_{1}^{ \pm}}=85 \mathrm{GeV}$} \\
\hline & \multicolumn{2}{|c|}{$\begin{array}{ll}\mathrm{LH} \\
\end{array}$} & \multicolumn{2}{|c|}{ 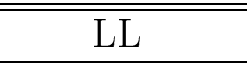 } & \multicolumn{2}{|c|}{$\overline{\mathrm{HH}}$} & \multicolumn{2}{|c|}{ 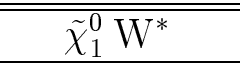 } \\
\hline$\Delta M(\mathrm{GeV})$ & $\epsilon(\%)$ & $N_{e x p}$ & $\epsilon(\%)$ & $N_{e x p}$ & $\epsilon(\%)$ & $N_{\text {exp }}$ & $\epsilon(\%)$ & $N_{\exp }$ \\
\hline 5 & 4.2 & 0.5 & 12 & 0.4 & 4.1 & 0.3 & 3.8 & 0.8 \\
\hline 10 & 40 & 0.3 & 33 & 0.4 & 38 & 0.3 & 29 & 0.3 \\
\hline 20 & 65 & 0.2 & 44 & 0.2 & 66 & 0.2 & 55 & 0.3 \\
\hline 30 & 73 & 0.2 & 57 & 0.3 & 60 & 0.3 & 52 & 0.3 \\
\hline 40 & 70 & 0.3 & 58 & 0.6 & 30 & 0.9 & 42 & 1.1 \\
\hline 60 & 62 & 0.7 & 27 & 0.6 & 23 & 0.2 & 32 & 0.8 \\
\hline 75 & 21 & 0.8 & 20 & 0.5 & 6.2 & 0.2 & 13 & 1.3 \\
\hline
\end{tabular}

Table 5: Chargino efficiencies $(\epsilon)$ for the lepton plus hadrons (LH), the purely leptonic (LL) and the purely hadronic (HH) final states and assuming $100 \% \tilde{\chi}_{1}^{0} \mathrm{~W}^{*}$ decay mode. $N_{\text {exp }}$ is the number of events expected from Standard Model processes. Results are given as a function of $\Delta M$ for $M_{\tilde{\chi}_{1}^{ \pm}}=80(85) \mathrm{GeV}$ pair production at $\sqrt{s}=161(172) \mathrm{GeV}$. 


\begin{tabular}{|c|c|c|c|c|c|c|}
\hline \multicolumn{3}{|c|}{$M_{\tilde{\chi}_{1}^{0}}+M_{\tilde{\chi}_{2}^{0}}=160 \mathrm{GeV}$} & \multicolumn{4}{|c|}{$\sqrt{s}=161 \mathrm{GeV}$} \\
\hline & \multicolumn{2}{|c|}{$\overline{\mathrm{HH}}$} & \multicolumn{2}{|c|}{ 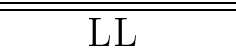 } & \multicolumn{2}{|c|}{$\tilde{\chi}_{1}^{0} Z^{*}$} \\
\hline$\Delta M(\mathrm{GeV})$ & $\epsilon(\%)$ & $N_{\text {exp }}$ & $\epsilon(\%)$ & $N_{\text {exp }}$ & $\epsilon(\%)$ & $N_{\text {exp }}$ \\
\hline 10 & 13 & 0.5 & 7.2 & 0.4 & 10 & 0.5 \\
\hline 20 & 40 & 0.6 & 12 & 0.5 & 27 & 0.4 \\
\hline 30 & 51 & 0.4 & 38 & 0.3 & 40 & 0.5 \\
\hline 40 & 52 & 0.3 & 47 & 0.6 & 36 & 0.3 \\
\hline 50 & 57 & 0.3 & 27 & 0.8 & 41 & 0.5 \\
\hline 70 & 54 & 0.8 & 31 & 0.5 & 40 & 0.8 \\
\hline 80 & 31 & 0.8 & 36 & 1.0 & 24 & 0.8 \\
\hline 120 & 2.6 & 0.6 & 10 & 0.9 & 2.8 & 1.4 \\
\hline \multicolumn{7}{|c|}{$M_{\tilde{\chi}_{1}^{0}}+M_{\tilde{\chi}_{2}^{0}}=170 \mathrm{GeV}$} \\
\hline & \multicolumn{2}{|c|}{$\overline{\mathrm{HH}}$} & \multicolumn{2}{|c|}{ LL } & \multicolumn{2}{|c|}{$\tilde{\chi}_{1}^{0} \mathrm{Z}^{*}$} \\
\hline$\Delta M(\mathrm{GeV})$ & $\epsilon(\%)$ & $N_{\text {exp }}$ & $\epsilon(\%)$ & $N_{\text {exp }}$ & $\epsilon(\%)$ & $N_{\text {exp }}$ \\
\hline 10 & 14 & 0.5 & 32 & 0.4 & 13 & 0.6 \\
\hline 20 & 52 & 0.4 & 42 & 0.5 & 40 & 0.5 \\
\hline 30 & 46 & 0.4 & 31 & 0.7 & 27 & 0.4 \\
\hline 40 & 39 & 0.4 & 37 & 0.8 & 26 & 0.3 \\
\hline 50 & 52 & 0.4 & 51 & 0.4 & 41 & 0.4 \\
\hline 70 & 50 & 0.7 & 35 & 0.3 & 37 & 0.8 \\
\hline 90 & 26 & 0.5 & 35 & 1.8 & 18 & 0.5 \\
\hline 130 & 8.9 & 2.3 & 29 & 2.3 & 9.1 & 2.4 \\
\hline
\end{tabular}

Table 6: Neutralino efficiencies $(\epsilon)$ for the purely hadronic $(\mathrm{HH})$, the purely leptonic (LL) final states and assuming $100 \% \tilde{\chi}_{1}^{0} \mathrm{Z}^{*}$ decay mode. Results are given as a function of $\Delta M$ for $M_{\tilde{\chi}_{2}^{0}}+M_{\tilde{\chi}_{1}^{0}}=160(170) \mathrm{GeV}$ at $\sqrt{s}=161(172) \mathrm{GeV}$.

\begin{tabular}{|c|c|c|}
\hline & $N_{\text {data }}$ & $N_{\text {exp }}$ \\
\hline$\tilde{\mathrm{e}}^{ \pm}$ & 3 & 1.3 \\
\hline$\tilde{\mu}^{ \pm}$ & 0 & 1.3 \\
\hline$\tilde{\tau}^{ \pm}$ & 1 & 1.2 \\
\hline$\tilde{\chi}_{1}^{ \pm}, \tilde{\chi}_{1}^{0}$ & 5 & 4.5 \\
\hline \hline Overall & 8 & 7.6 \\
\hline
\end{tabular}

Table 7: Results on scalar leptons, chargino and neutralino searches: $N_{\text {data }}$ is the number of observed events and $N_{\text {exp }}$ is the number of expected events from Standard Model processes for the total integrated luminosities recorded at $\sqrt{s}=161 \mathrm{GeV}$ and $\sqrt{s}=172 \mathrm{GeV}$. 

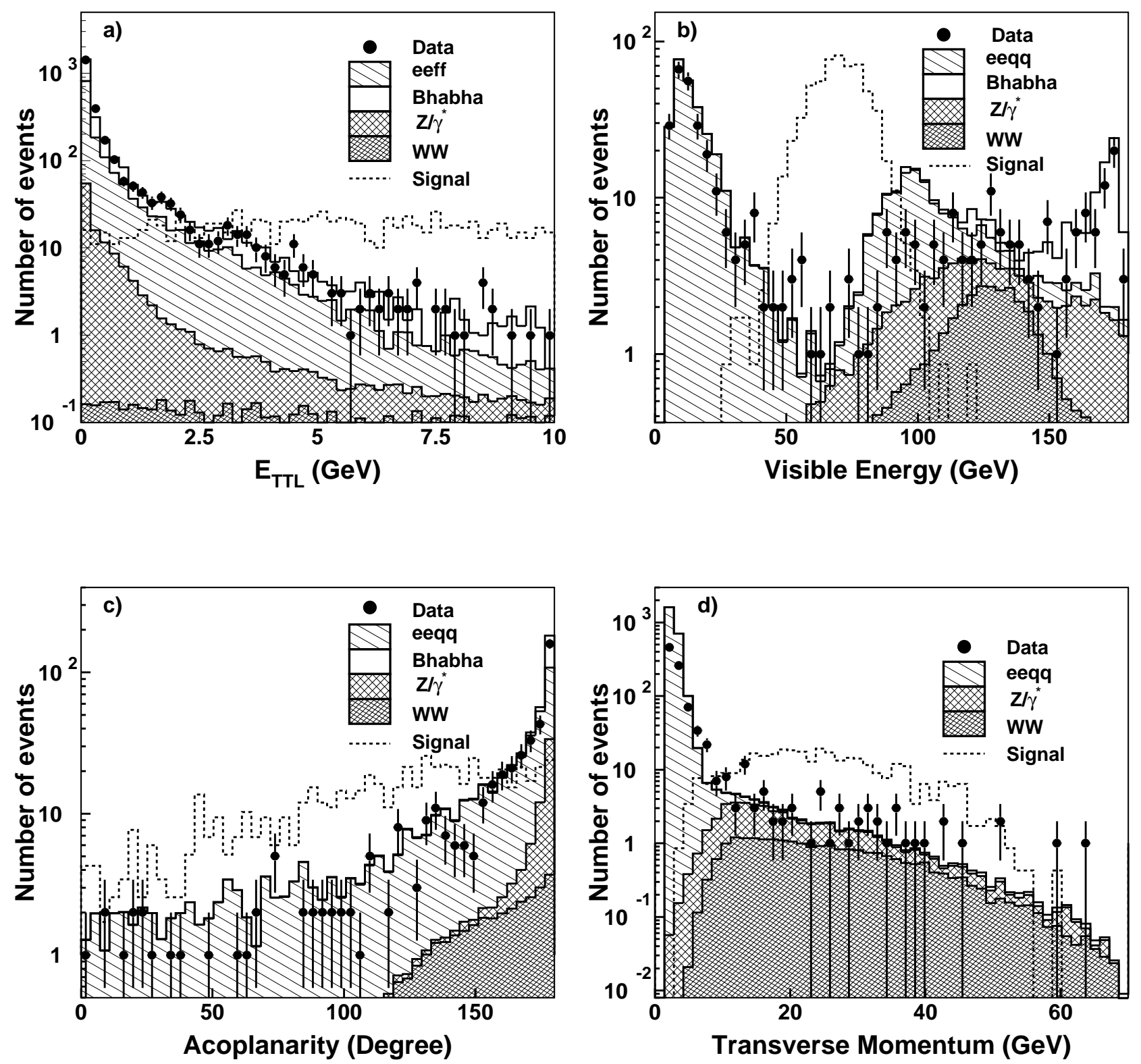

Figure 1: Distributions illustrating the selection of signal events at $\sqrt{s}=172 \mathrm{GeV}$. Data are compared to Standard Model background processes (hatched histograms) and to the expected signal (open histograms): a) the variable $\mathrm{E}_{T T L}$ (see section 3.2.1) for purely leptonic final states. b) Visible energy and c) acoplanarity of lepton plus hadron final states. d) The distribution of the transverse momentum in purely hadronic final states. The signal distributions are evaluated for $M_{\tilde{\chi}_{1}^{ \pm}}=85 \mathrm{GeV}$ and $M_{\tilde{\chi}_{1}^{0}}=30 \mathrm{GeV}$ for a) and $M_{\tilde{\chi}_{1}^{0}}=50 \mathrm{GeV}$ for b) $-\mathrm{d}$ ). 


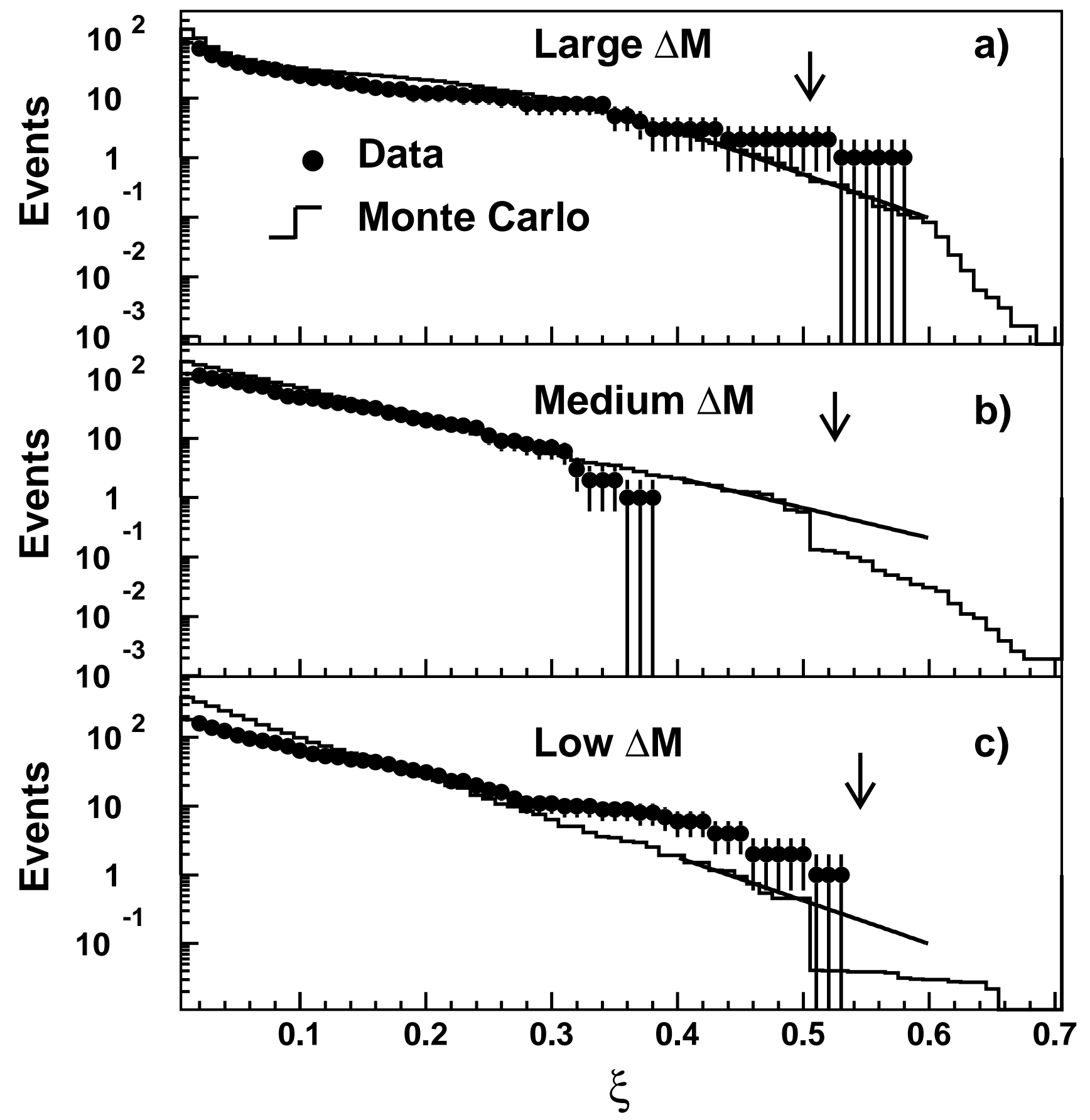

Figure 2: Number of events selected in data and in Monte Carlo of Standard Model processes as a function of selection cuts with increasing background rejection. The vertical arrows show the position of the optimized cuts. The smooth lines correspond to the analytical extrapolation for the background estimation. a), b) and c) show the distributions for the large, medium and low $\Delta M$ selections of purely hadronic final states, respectively. 

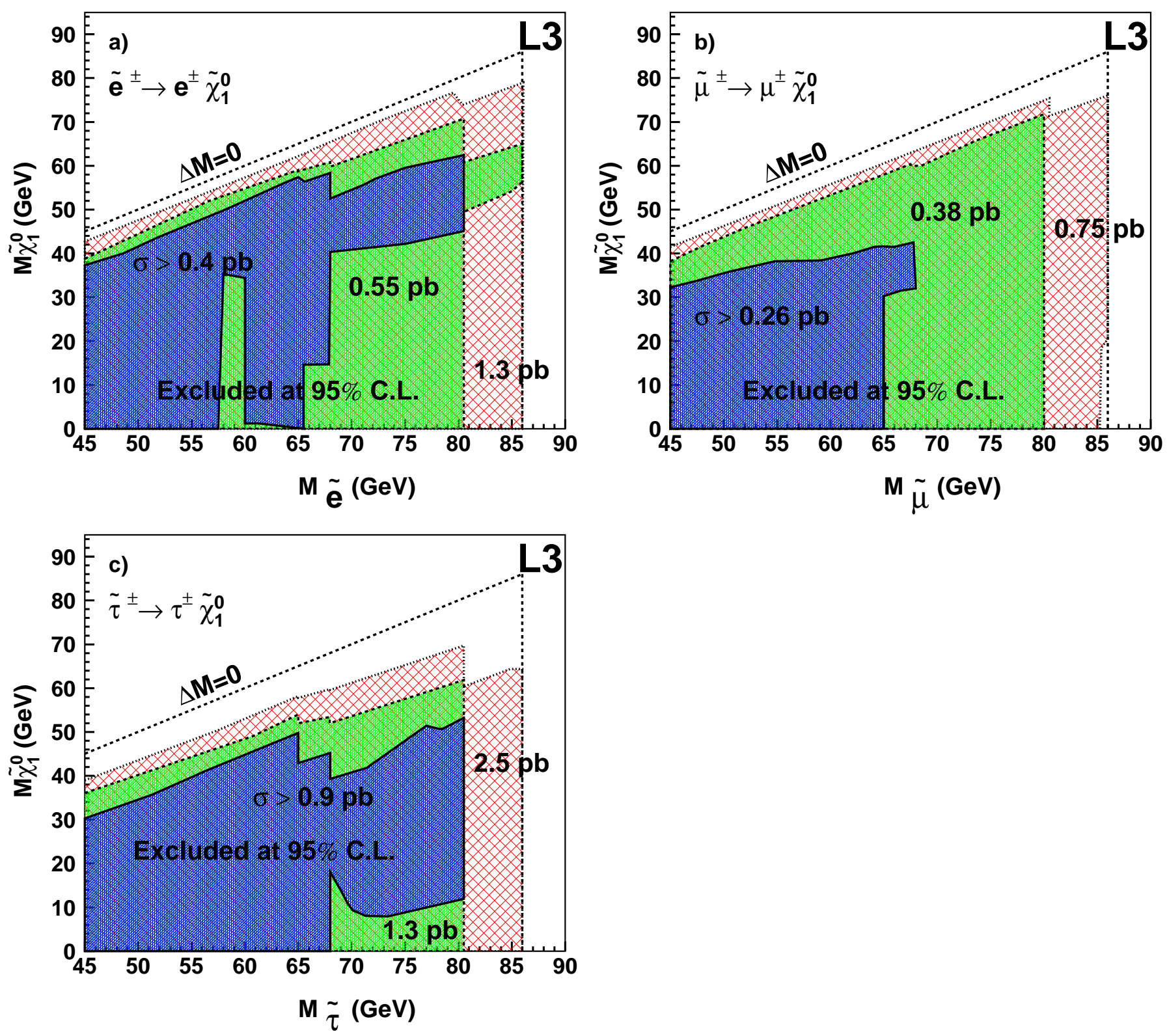

Figure 3: Upper limits on the production cross sections for scalar leptons as a function of the lightest neutralino mass $M_{\tilde{\chi}_{1}^{0}}$. a) - c) show the limits for scalar electrons, muons and taus, respectively. 

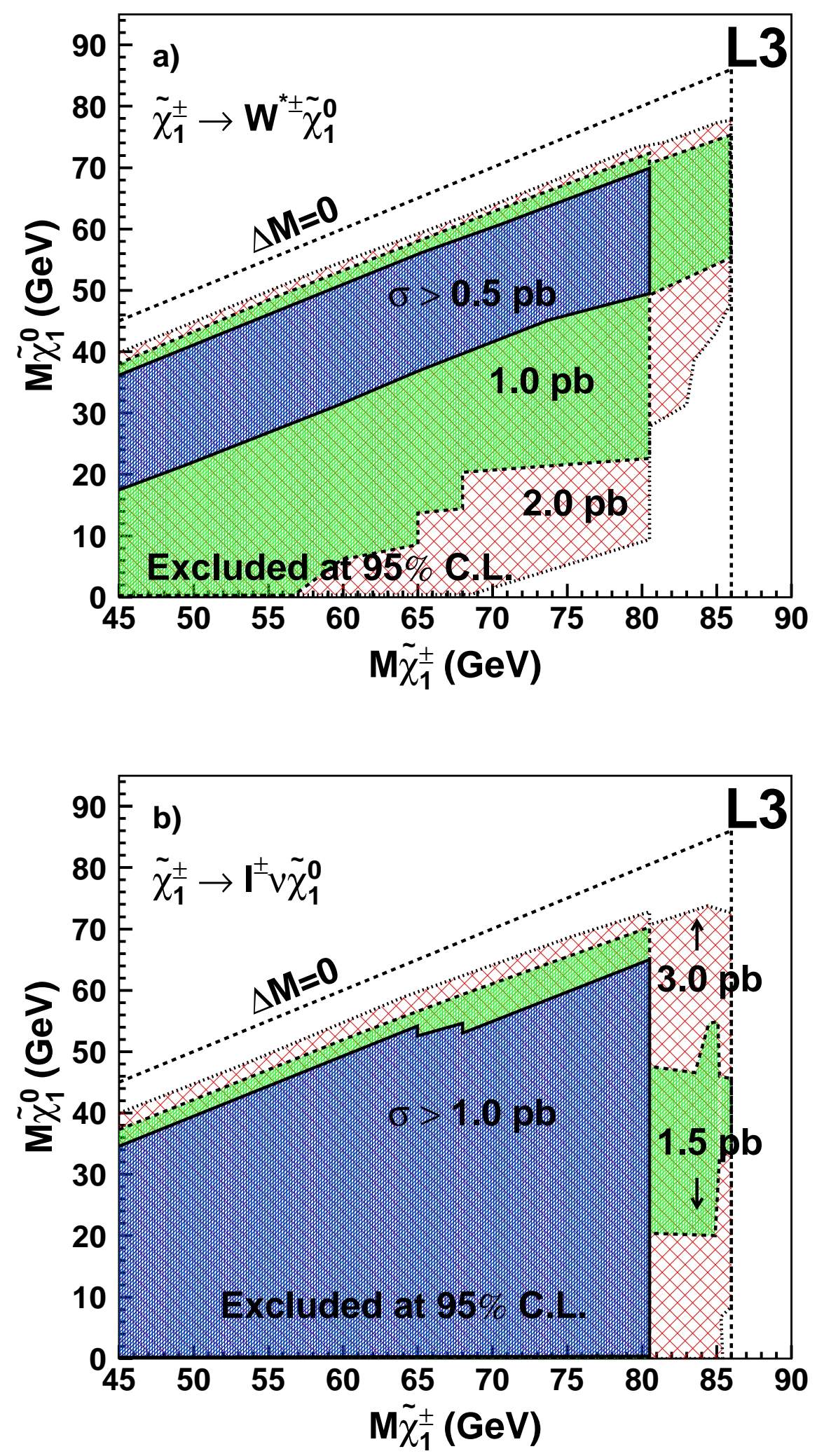

Figure 4: Upper limits on the production cross section $\mathrm{e}^{+} \mathrm{e}^{-} \rightarrow \tilde{\chi}_{1}^{+} \tilde{\chi}_{1}^{-}$in the $M_{\tilde{\chi}_{1}^{0}}-M_{\tilde{\chi}_{1}^{ \pm}}$plane. a) Exclusion limits are obtained assuming standard $W$ branching ratios in the chargino decay $\tilde{\chi}_{1}^{ \pm} \rightarrow \mathrm{W}^{* \pm} \tilde{\chi}_{1}^{0}$ and b) assuming purely leptonic $\mathrm{W}$ decays $\tilde{\chi}_{1}^{ \pm} \rightarrow \ell^{ \pm} \nu \tilde{\chi}_{1}^{0},(\ell=\mathrm{e}, \mu, \tau)$. 


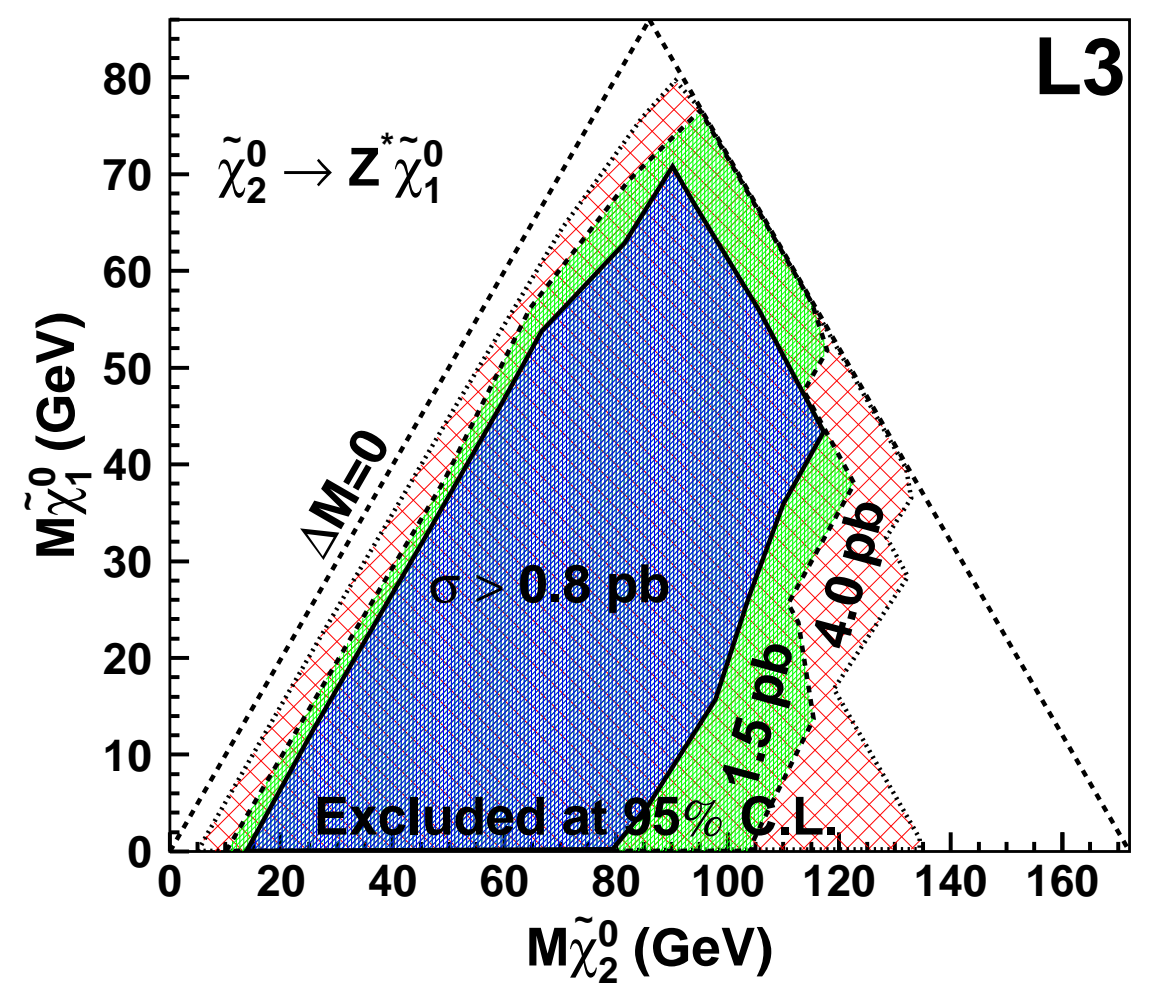

Figure 5: Upper limits on the production cross section of $\mathrm{e}^{+} \mathrm{e}^{-} \rightarrow \tilde{\chi}_{1}^{0} \tilde{\chi}_{2}^{0}$ in the $M_{\tilde{\chi}_{1}^{0}}-M_{\tilde{\chi}_{2}^{0}}$ plane. Exclusion limits are obtained assuming standard $\mathrm{Z}^{*}$ branching ratios in the neutralino decay $\tilde{\chi}_{2}^{0} \rightarrow \mathrm{Z}^{* \pm} \tilde{\chi}_{1}^{0}$. 

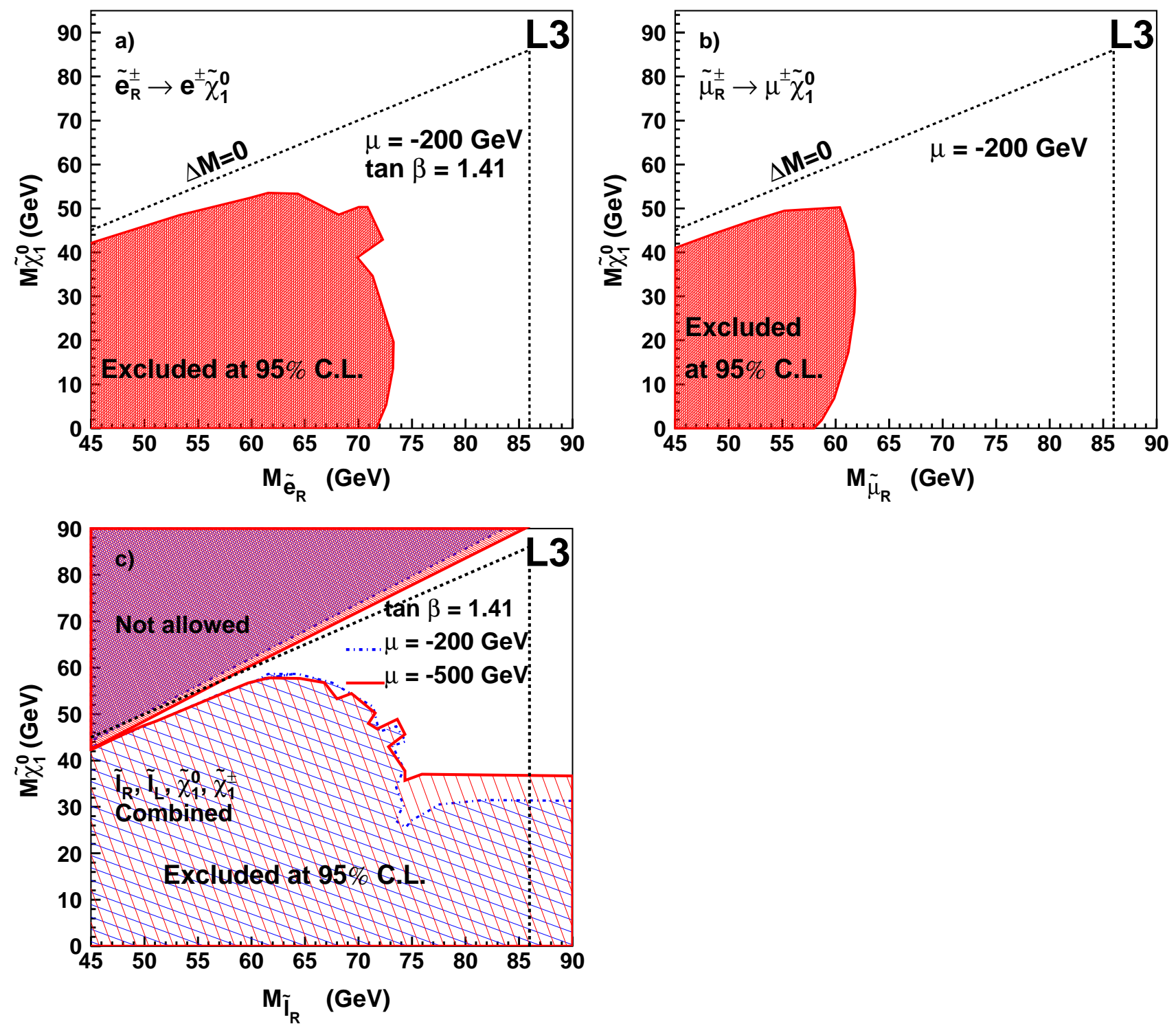

Figure 6: Mass limits on the scalar partners of right-handed electrons a) and muons b) as a function of the neutralino mass $M_{\tilde{\chi}_{1}^{0}}$. These two figures are obtained using only the upper limits on the cross section obtained from direct searches. c) shows the limits for scalar leptons assuming universality for scalar masses. The latter is derived from the results of the searches for scalar leptons, charginos and neutralinos in the constrained MSSM. In the upper left corner, regions not allowed in the MSSM are indicated. 

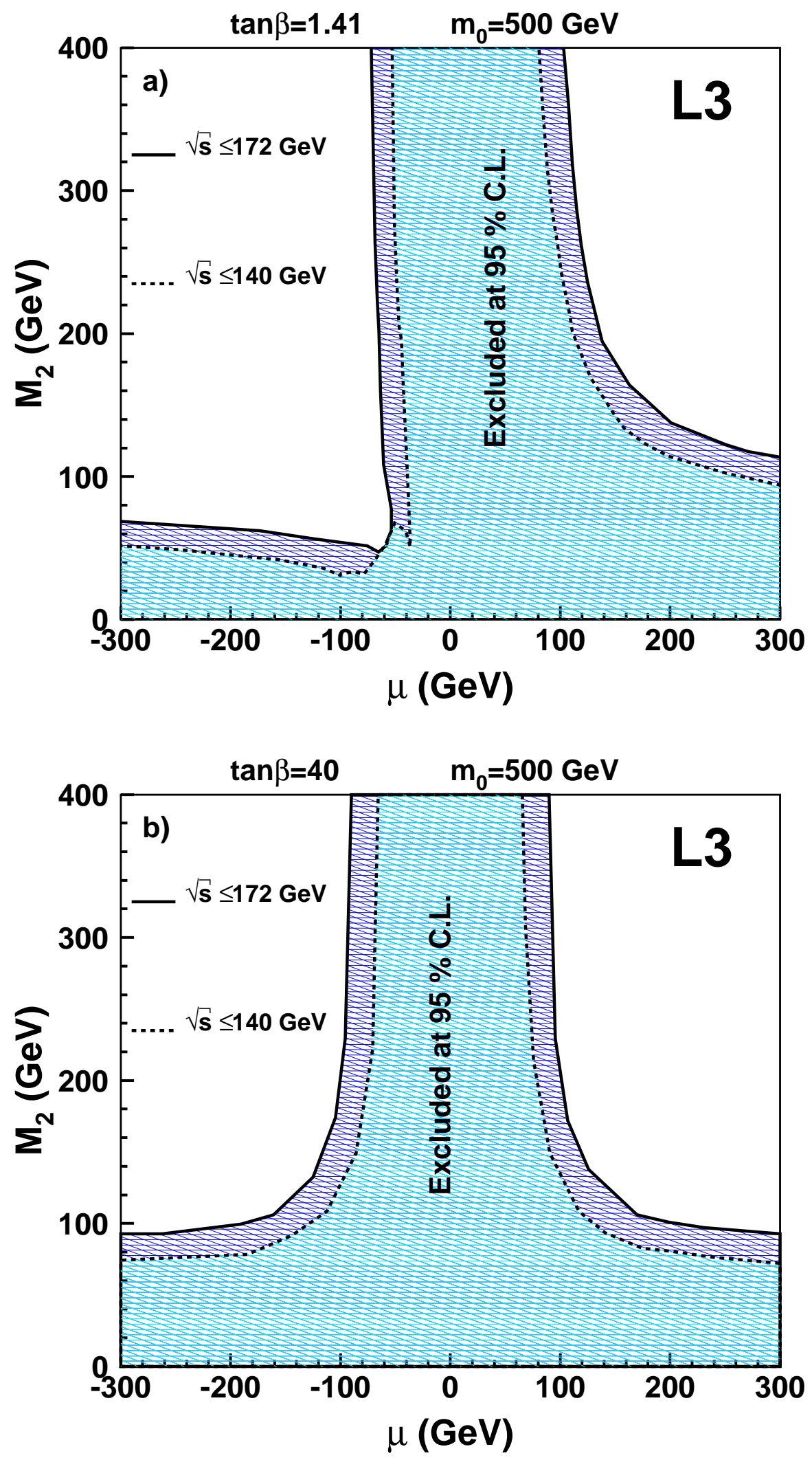

Figure 7: Exclusion regions in the $M_{2}-\mu$ plane derived from the search for charginos and neutralinos for $m_{0}=500 \mathrm{GeV}$ with $\tan \beta=1.41 \mathrm{a}$ ) and $40 \mathrm{~b}$ ). The results using all data (solid lines) are compared to the limits obtained using only data collected at the $\mathrm{Z}$ resonance and at $\sqrt{s}=130-140 \mathrm{GeV}$ (broken lines). 

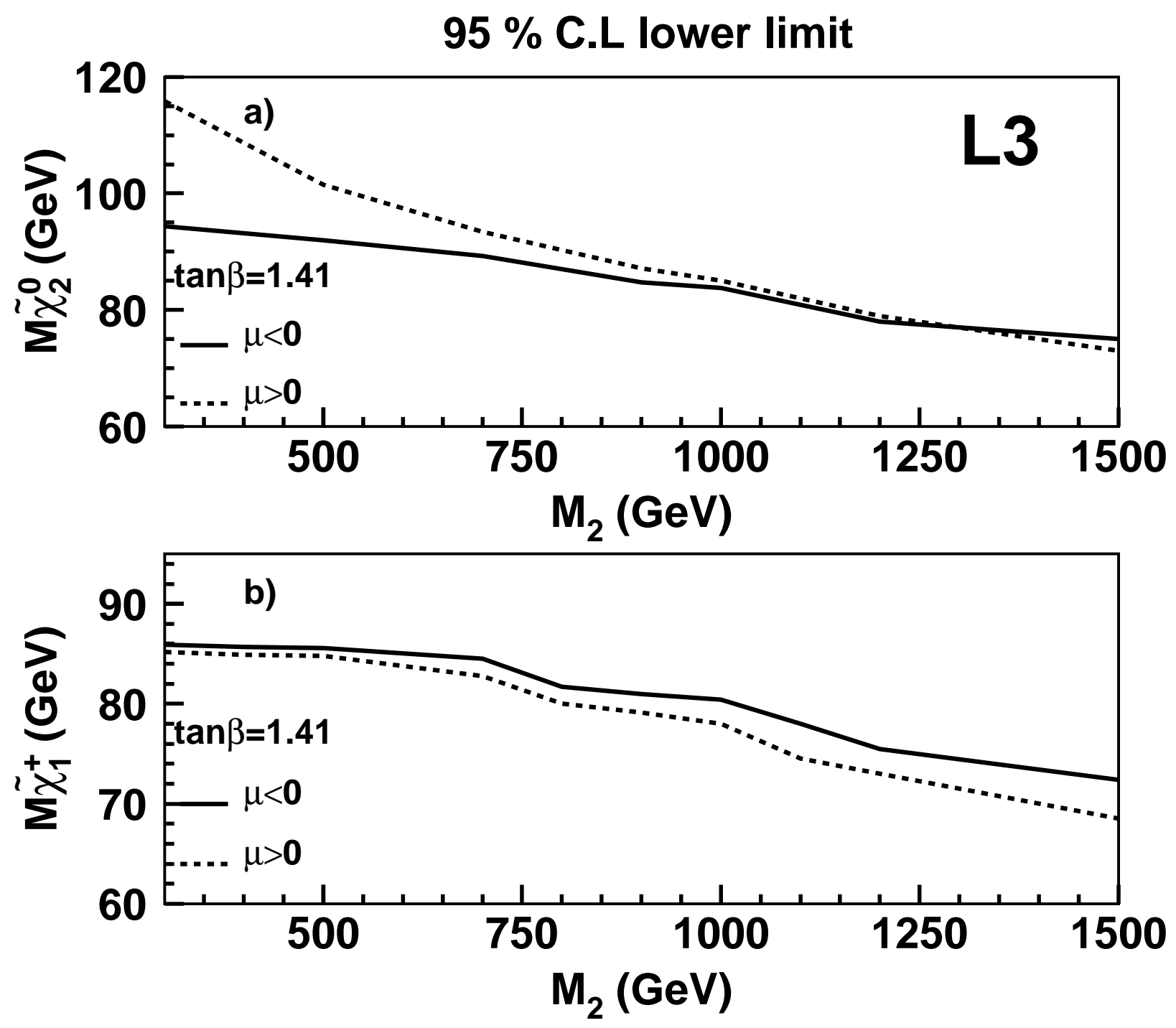

Figure 8: Mass limits as a function of $M_{2}$ for the next to lightest neutralino $\tilde{\chi}_{2}^{0}$ a) and the lightest chargino $\tilde{\chi}_{1}^{ \pm} \mathrm{b}$ ). The limits are obtained for $\tan \beta=1.41$ and for $\mu<0$ and $\mu>0$ (solid and broken lines, respectively). 


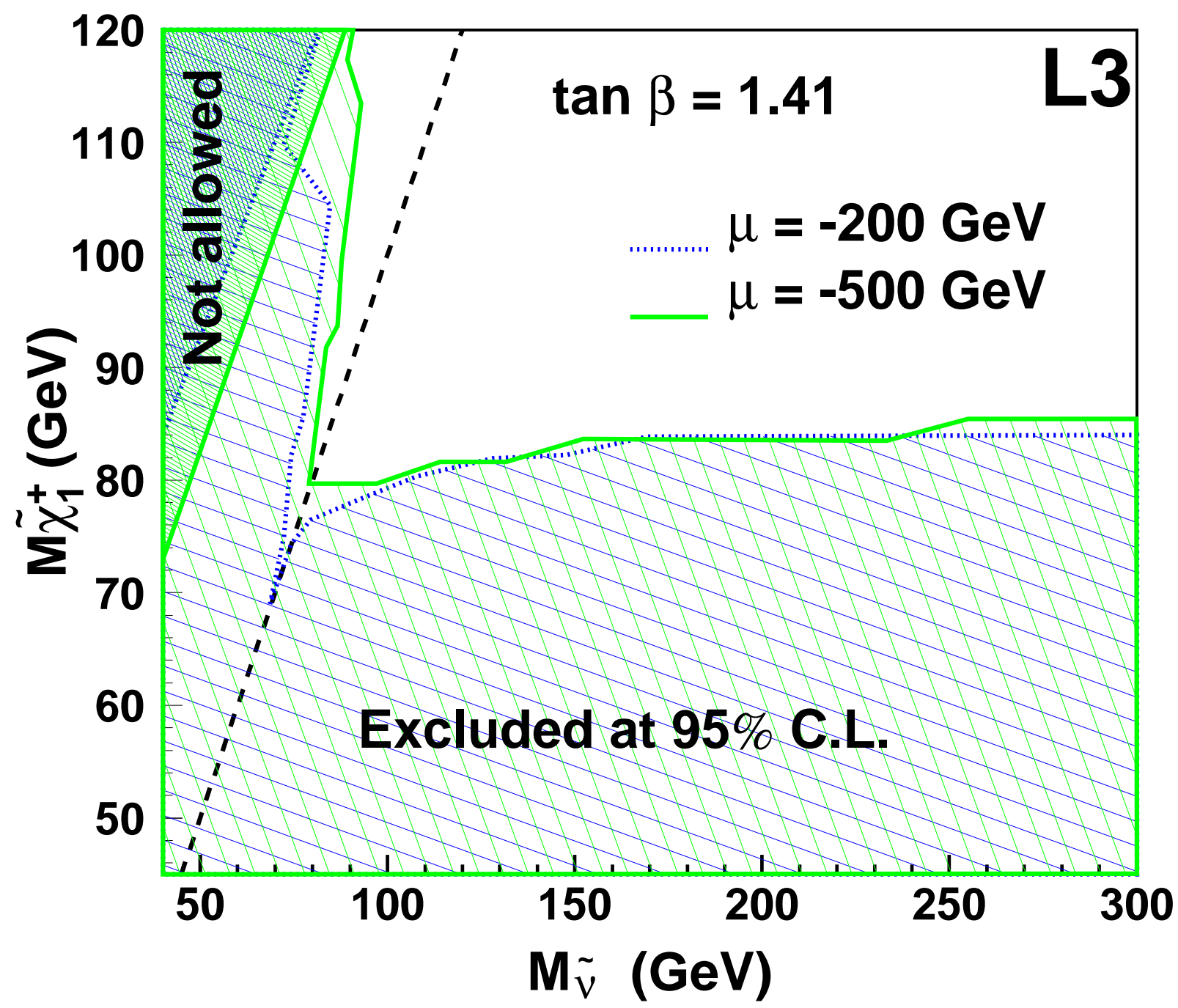

Figure 9: Mass limits for the chargino as a function of the scalar neutrino mass for $\mu=-500$ $\mathrm{GeV}$ (solid line) and $\mu=-200 \mathrm{GeV}$ (dotted line), for $\tan \beta=1.41$. The dashed line corresponds to $M_{\tilde{\chi}_{1}^{ \pm}}=M_{\tilde{\nu}}$. The region in the upper left corner is not allowed in the MSSM. 

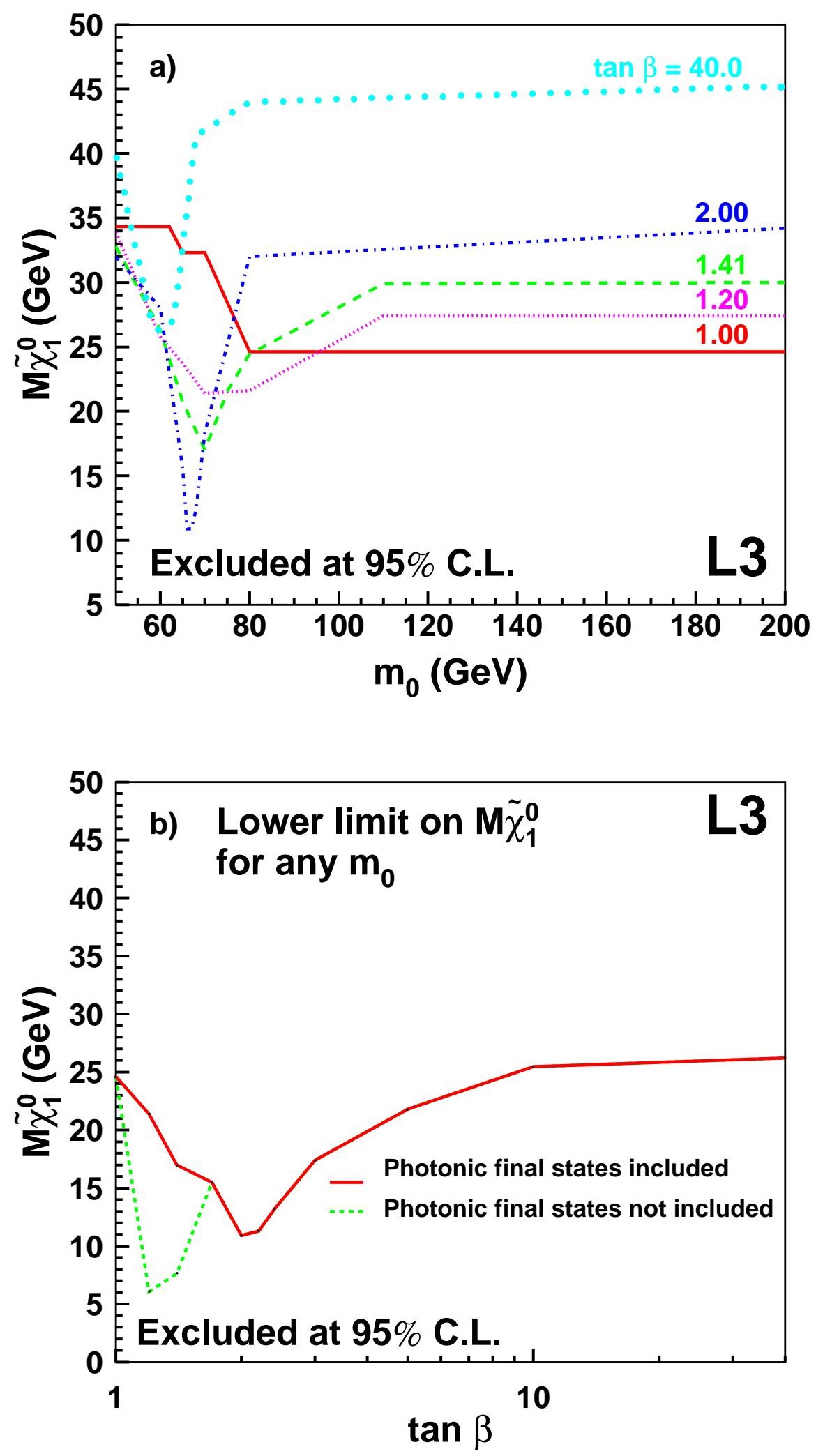

Figure 10: a) Lower limit on the lightest neutralino mass $M_{\tilde{\chi}_{1}^{0}}$ as a function of $m_{0}$ for five different values of $\tan \beta$. b) Lower limit on $M_{\tilde{\chi}_{1}^{0}}$ as a function of $\tan \beta$ and for any value of $m_{0}$. 